\title{
Quantum gravity on the lattice
}

\author{
Herbert W. Hamber
}

Received: 31 December 2008 / Accepted: 19 January 2009 / Published online: 8 February 2009

(C) The Author(s) 2009. This article is published with open access at Springerlink.com

\begin{abstract}
I review the lattice approach to quantum gravity, and how it relates to the non-trivial ultraviolet fixed point scenario of the continuum theory. After a brief introduction covering the general problem of ultraviolet divergences in gravity and other non-renormalizable theories, I discuss the general methods and goals of the lattice approach. An underlying theme is the attempt at establishing connections between the continuum renormalization group results, which are mainly based on diagrammatic perturbation theory, and the recent lattice results, which apply to the strong gravity regime and are inherently non-perturbative. A second theme in this review is the ever-present natural correspondence between infrared methods of strongly coupled non-abelian gauge theories on the one hand, and the low energy approach to quantum gravity based on the renormalization group and universality of critical behavior on the other. Towards the end of the review I discuss possible observational consequences of path integral quantum gravity, as derived from the non-trivial ultraviolet fixed point scenario. I argue that the theoretical framework naturally leads to considering a weakly scale-dependent Newton's constant, with a scaling violation parameter related to the observed scaled cosmological constant (and not, as naively expected, to the Planck length).
\end{abstract}

Keywords Quantum gravity - Covariant quantization · Feynman path integral · Lattice gravity · Quantum cosmology

Invited lecture presented at the conference "Quantum Gravity: Challenges and Perspectives", Bad Honnef, 14-16 April 2008. To appear in the proceedings edited by Hermann Nicolai.

H. W. Hamber $(\bowtie)$

Department of Physics, University of California, Irvine, CA 92717, USA

e-mail: hhamber@uci.edu 


\section{Introduction and motivation}

Since the seventies strategies that deal with the problem of ultraviolet divergences in quantum gravity have themselves diverged. Some have advocated the search for a new theory of quantum gravity, a theory which does not suffer from ultraviolet infinity problems. In supersymmetric theories, such as supergravity and ten-dimensional superstrings, new and yet unobserved particles are introduced thus reducing the divergence properties of Feynman amplitudes. In other, very restricted classes of supergravity theories in four dimensions, proponents have claimed that enough conspiracies might arise thereby making these models finite. For superstrings, which live in a ten-dimensional spacetime, one major obstacle prevails to date: what dynamical mechanism would drive the compactification of spacetime from the ten dimensional string universe to our physical four-dimensional world?

A second approach to quantum gravity has endeavored to pursue new avenues to quantization, by introducing new quantum variables and new cutoffs, which involve quantum Hamiltonian methods based on parallel transport loops, spacetime spin foam and new types of quantum variables describing a quantum dust. It is characteristic of these methods that the underlying theory is preserved: it essentially remains a quantum version of Einstein's relativistic theory, yet the ideas employed are intended to go past the perturbative treatment. While some of these innovative tools have had limited success in exploring the much simpler non-perturbative features of ordinary gauge theories, proponents of such methods have argued that gravity is fundamentally different, thereby necessitating the use of radically new methods.

The third approach to quantum gravity, which forms the topic of this review, focuses instead on the application of modern methods of quantum field theory. Its cornerstones include the manifestly covariant Feynman path integral approach, Wilson's modern renormalization group ideas and the development of lattice methods to define a regularized form of the path integral, which would then allow controlled non-perturbative calculations. In non-abelian gauge theories and in the standard model of elementary particle interactions, these methods are invariably the tools of choice: the covariant Feynman path integral approach is crucial in proving the renormalizability of nonabelian gauge theories; modern renormalization group methods establish the core of the derivation of the asymptotic freedom result and related discussions of momentum dependence of amplitudes in terms of a running coupling constant; and finally, the lattice formulation of gauge theories, which so far provides the only convincing theoretical evidence of confinement and chiral symmetry breaking in non-abelian gauge theories.

\section{Ultraviolet divergences and perturbative non-renormalizability}

In gravity the coupling is dimensionful, $G \sim \mu^{2-d}$, and one expects trouble in four dimensions already on purely dimensional grounds, with divergent one loop corrections proportional to $G \Lambda^{d-2}$ where $\Lambda$ is the ultraviolet cutoff. Phrased differently, one expects to lowest order some seriously bad ultraviolet behavior from the running of Newton's constant at large momenta, 


$$
G\left(k^{2}\right) / G \sim 1+\text { const. } G k^{d-2}+O\left(G^{2}\right)
$$

While problematic in four dimensions, these considerations also suggest that ordinary Einstein gravity should be perturbatively renormalizable in the traditional sense in two dimensions, an issue to which we will return later.

The more general argument for perturbative non-renormalizability goes as follows. The gravitational action contains the scalar curvature $R$ which involves two derivatives of the metric. Thus the graviton propagator in momentum space will go like $1 / k^{2}$, and the vertex functions like $k^{2}$. In $d$ dimensions each loop integral with involve a momentum integration $d^{d} k$, so that the superficial degree of divergence $\mathcal{D}$ of a Feynman diagram with $L$ loops will be given by

$$
\mathcal{D}=2+(d-2) L
$$

independent of the number of external lines. One concludes that for $d>2$ the degree of ultraviolet divergence increases with increasing loop order $L$.

The most convenient tool to determine the structure of the divergent one-loop corrections to Einstein gravity is the background field method combined with dimensional regularization, wherein ultraviolet divergences appear as poles in $\epsilon=d-4$. In nonAbelian gauge theories the background field method greatly simplifies the calculation of renormalization factors, while at the same time maintaining explicit gauge invariance. The essence of the method is easy to describe: one replaces the original field appearing in the classical action by $A+Q$, where $A$ is a classical background field and $Q$ the quantum fluctuation. A suitable gauge condition is chosen (the background gauge), such that manifest gauge invariance is preserved for the background $A$ field. After expanding out the action to quadratic order in the $Q$ field, the functional integration over $Q$ is performed, leading to an effective action for the background $A$ field. This method eventually determines, after a rather lengthy calculation, the required one-loop counterterm for pure gravity

$$
\Delta \mathcal{L}_{g}=\frac{\sqrt{g}}{8 \pi^{2}(d-4)}\left(\frac{1}{120} R^{2}+\frac{7}{20} R_{\mu \nu} R^{\mu \nu}\right) .
$$

There are two interesting, and interrelated, aspects of the result of (3). The first one is that for pure gravity the divergent part vanishes when one imposes the tree-level equations of motion $R_{\mu \nu}=0$ : the one-loop divergence vanishes on-shell. The second interesting aspect is that the specific structure of the one-loop divergence is such that its effect can actually be re-absorbed into a field redefinition,

$$
g_{\mu \nu} \rightarrow g_{\mu \nu}+\delta g_{\mu \nu} \quad \delta g_{\mu \nu} \propto \frac{7}{20} R_{\mu \nu}-\frac{11}{60} R g_{\mu \nu}
$$

which renders the one-loop amplitudes finite for pure gravity. It appears though that these two aspects are largely coincidental; unfortunately this hoped-for mechanism does not seem to work to two loops, and no additional miraculous cancellations seem to occur there. 
One can therefore attempt to summarize the (perturbative) situation so far as follows: In principle perturbation theory in $G$ in provides a clear, covariant framework in which radiative corrections to gravity can be computed in a systematic loop expansion. The effects of a possibly non-trivial gravitational measure do not show up at any order in the weak field expansion, and radiative corrections affecting the renormalization of the cosmological constant, proportional to $\delta^{d}(0)$, are set to zero in dimensional regularization.

At the same time, at every order in the loop expansion new invariant terms involving higher derivatives of the metric are generated, whose effects cannot simply be absorbed into a re-definition of the original couplings. As expected on the basis of power-counting arguments, the theory is not perturbatively renormalizable in the traditional sense in four dimensions (although it seems to fail this test by a small measure in lowest order perturbation theory).

Thus the standard approach based on a perturbative expansion of the pure Einstein theory in four dimensions is clearly not convergent (it is in fact badly divergent), and represents therefore a temporary dead end. The key question is therefore if this is an artifact of naive perturbation theory, or not.

\section{Feynman path integral for quantum gravitation}

If non-perturbative effects play an important role in quantum gravity, then one would expect the need for an improved formulation of the quantum theory is, which would not rely exclusively on the framework of a perturbative expansion. After all, the fluctuating metric field $g_{\mu \nu}$ is dimensionless, and carries therefore no natural scale. For the somewhat simpler cases of a scalar field and non-Abelian gauge theories a consistent non-perturbative formulation based on the Feynman path integral has been known for some time, and is by now well developed. In a nutshell, the Feynman path integral formulation for pure quantum gravitation can be expressed in the functional integral formula

$$
Z=\int_{\text {geometries }} e^{\frac{i}{\hbar} I_{\text {geometry }}}
$$

just like the Feynman path integral for a non-relativistic quantum mechanical particle expresses quantum-mechanical amplitudes in terms of sums over paths

$$
A(i \rightarrow f)=\int_{\text {paths }} e^{\frac{i}{\hbar} I_{\text {path }}}
$$

What is the precise meaning of the expression in (5)? In the case of quantum fields, one is generally interested in a vacuum-to-vacuum amplitude, which requires $t_{i} \rightarrow-\infty$ and $t_{f} \rightarrow+\infty$. For a scalar field the functional integral with sources is generally of the form 


$$
Z[J]=\int[d \phi] \exp \left\{i \int d^{4} x[\mathcal{L}(x)+J(x) \phi(x)]\right\}
$$

where $[d \phi]=\prod_{x} d \phi(x)$, and $\mathcal{L}$ the usual Lagrangian density for a scalar field. It is important to note that even with an underlying lattice discretization, the integral in (7) is in general ill-defined without a damping factor, due to the overall $i$ in the exponent. Advances in axiomatic field theory indicate that if one is able to construct a well defined field theory in Euclidean space $x=(\mathbf{x}, \tau)$ obeying certain axioms, then there is a corresponding field theory in Minkowski space $(\mathbf{x}, t) t=-i \tau$ defined as an analytic continuation of the Euclidean theory, such that it obeys the Wightmann axioms.

Turning to the case of gravity, it should be clear that at least to all orders in the weak field expansion there is really no difference of substance between the Lorentzian (or pseudo-Riemannian) and the Euclidean (or Riemannian) formulation. Indeed most, if not all, of the perturbative calculations of the preceding section could have been carried out with the Riemannian weak field expansion about flat Euclidean space $g_{\mu \nu}=\delta_{\mu \nu}+h_{\mu \nu}$ with signature ++++ , or about some suitable classical Riemannian background manifold. Now in function space one needs a metric before one can define a volume element. Therefore, following DeWitt, one needs first to define an invariant norm for metric deformations

$$
\|\delta g\|^{2}=\int d^{d} x \delta g_{\mu \nu}(x) G^{\mu \nu, \alpha \beta}(g(x)) \delta g_{\alpha \beta}(x),
$$

with the supermetric $G$ given by the ultra-local expression

$$
G^{\mu \nu, \alpha \beta}(g(x))=\frac{1}{2} \sqrt{g(x)}\left[g^{\mu \alpha}(x) g^{\nu \beta}(x)+g^{\mu \beta}(x) g^{\nu \alpha}(x)+\lambda g^{\mu \nu}(x) g^{\alpha \beta}(x)\right]
$$

with $\lambda$ a real parameter, $\lambda \neq-2 / d$. The DeWitt supermetric then defines a suitable volume element $\sqrt{G}$ in function space, such that the functional measure over the $g_{\mu \nu}$ 's taken on the form

$$
\int\left[d g_{\mu \nu}\right] \equiv \int \prod_{x}[\operatorname{det} G(g(x))]^{1 / 2} \prod_{\mu \geq \nu} d g_{\mu \nu}(x) .
$$

Thus the local measure for the Feynman path integral for pure gravity is given by

$$
\int \prod_{x}[g(x)]^{(d-4)(d+1) / 8} \prod_{\mu \geq v} d g_{\mu \nu}(x)
$$

In four dimensions this becomes simply

$$
\int\left[d g_{\mu \nu}\right]=\int \prod_{x} \prod_{\mu \geq v} d g_{\mu \nu}(x)
$$


However it is not obvious that the above construction is unique. A more general measure would contain the additional volume factor $g^{\sigma / 2}$ in a slightly more general gravitational functional measure

$$
\int\left[d g_{\mu \nu}\right]=\prod_{x}[g(x)]^{\sigma / 2} \prod_{\mu \geq v} d g_{\mu \nu}(x)
$$

Therefore it is important in this context that one can show that the gravitational functional measure of (13) is invariant under infinitesimal general coordinate transformations, irrespective of the value of $\sigma$.

So in conclusion, the Euclidean Feynman path integral for pure Einstein gravity with a cosmological constant term is given by

$$
Z_{\text {cont }}=\int\left[d g_{\mu \nu}\right] \exp \left\{-\lambda_{0} \int d x \sqrt{g}+\frac{1}{16 \pi G} \int d x \sqrt{g} R\right\} .
$$

Still not all is well. Euclidean quantum gravity suffers potentially from a disastrous problem associated with the conformal instability: the presence of kinetic contributions to the linearized action entering with the wrong sign. If one writes down a path integral for pure gravity in the form of (14) one realizes that it appears ill defined due to the fact that the scalar curvature can become arbitrarily positive. In turn this can be seen as related to the fact that while gravitational radiation has positive energy, gravitational potential energy is negative, because gravity is attractive. To see more clearly that the gravitational action can be made arbitrarily negative consider the conformal transformation $\tilde{g}_{\mu \nu}=\Omega^{2} g_{\mu \nu}$ where $\Omega$ is some positive function. Then the Einstein action transforms into

$$
I_{E}(\tilde{g})=-\frac{1}{16 \pi G} \int d^{4} x \sqrt{g}\left(\Omega^{2} R+6 g^{\mu \nu} \partial_{\mu} \Omega \partial_{\nu} \Omega\right) .
$$

which can be made arbitrarily negative by choosing a rapidly varying conformal factor $\Omega$. Indeed in the simplest case of a metric $g_{\mu \nu}=\Omega^{2} \eta_{\mu \nu}$ one has

$$
\sqrt{g}(R-2 \lambda)=6 g^{\mu \nu} \partial_{\mu} \Omega \partial_{\nu} \Omega-2 \lambda \Omega^{4}
$$

which looks like a $\lambda \phi^{4}$ theory but with the wrong sign for the kinetic term. The problem is referred to as the conformal instability of the classical Euclidean gravitational action.

A possible solution to the unboundedness problem of the Euclidean theory is that perhaps it should not be regarded as necessarily an obstacle to defining a quantum theory non-perturbatively. After all the quantum mechanical attractive Coulomb well problem has, for zero orbital angular momentum or in the one-dimensional case, a similar type of instability, since the action there is also unbounded from below. The way the quantum mechanical treatment ultimately evades the problem is that the particle has a vanishingly small probability amplitude to fall into the infinitely deep well. In quantum gravity the question regarding the conformal instability can then be rephrased 
in a similar way: will the quantum fluctuations in the metric be strong enough so that physical excitations will not fall into the conformal well? Of course to answer such questions satisfactorily one needs a formulation which is not restricted to small fluctuations, perturbation theory and the weak field limit. Ultimately in the lattice theory the answer seems yes, at least for sufficiently strong coupling $G$.

\section{Perturbatively non-renormalizable theories: the sigma model}

Einstein gravity is not perturbatively renormalizable in the traditional sense in four dimensions. Concretely this means that to one-loop order higher derivative terms are generated as radiative corrections with divergent coefficients. The natural question then arises: Are there any other field theories where the standard perurbative treatment fails, yet for which one can find alternative methods and from them develop consistent predictions? The answer seems unequivocally yes. Indeed outside of gravity, there are two notable examples of field theories, the non-linear sigma model and the self-coupled fermion model, which are not perturbatively renormalizable for $d>2$, and yet lead to consistent, and in some instances testable, predictions above $d=2$.

The key ingredient to all of these results is, as originally recognized by Wilson, the existence of a non-trivial ultraviolet fixed point (a phase transition in statistical field theory), with non-trivial universal scaling dimensions. Furthermore, one is lucky enough that for the non-linear $\sigma$-model three quite different theoretical approaches are available for comparing quantitative predictions: the $2+\epsilon$ expansion, the large- $N$ limit, and the lattice approach. Within the lattice approach, several additional techniques become available: the strong coupling expansion, the weak coupling expansion, real space renormalization group methods, and the numerically exact evaluation of the path integral. Finally, the results for the non-linear sigma model in the scaling regime around the non-trivial ultraviolet fixed point can be compared to recent high accuracy satellite (space shuttle) experiments on three-dimensional systems, and the results agree, the $O(2)$ non-linear $\sigma$-model in three dimensions, in some cases to several decimals, providing one of the most accurate tests of quantum field theory to date!

Concretely, the non-linear $\sigma$-model is a simple model describing the dynamics of an $N$-component field $\phi_{a}$ satisfying a unit constraint $\phi^{2}(x)=1$, and with functional integral given by

$$
Z[J]=\int[d \phi] \prod_{x} \delta[\phi(x) \cdot \phi(x)-1] \exp \left(-\frac{\Lambda^{d-2}}{g} S(\phi)+\int d^{d} x J(x) \cdot \phi(x)\right) .
$$

The action is taken to be $O(N)$-invariant

$$
S(\phi)=\frac{1}{2} \int d^{d} x \partial_{\mu} \phi(x) \cdot \partial_{\mu} \phi(x)
$$

$\Lambda$ here is the ultraviolet cutoff and $g$ the bare dimensionless coupling at the cutoff scale $\Lambda$; in a statistical field theory context $g$ plays the role of a temperature, and $\Lambda$ 
is proportional to the inverse lattice spacing. Above two dimensions, $d-2=\epsilon>0$ and a perturbative calculation determines the coupling renormalization. One finds for the effective coupling $g_{e}$ using dimensional regularization

$$
\frac{1}{g_{e}}=\frac{\Lambda^{\epsilon}}{g}\left[1-\frac{1}{\epsilon} \frac{N-2}{2 \pi} g+O\left(g^{2}\right)\right]
$$

This then gives immediately the Callan-Symanzik $\beta$-function for $g$

$$
\Lambda \frac{\partial g}{\partial \Lambda}=\beta(g)=\epsilon g-\frac{N-2}{2 \pi} g^{2}+O\left(g^{3}, \epsilon g^{2}\right)
$$

which determines the scale dependence of $g(\mu)$ for an arbitrary momentum scale $\mu$. The scale dependence of $g(\mu)$ is such that if the initial $g$ is less than the ultraviolet fixed point value $g_{c}$, with

$$
g_{c}=\frac{2 \pi \epsilon}{N-2}+\cdots
$$

then the coupling will flow towards the Gaussian fixed point at $g=0$. The new phase that appears when $\epsilon>0$ and corresponds to a low temperature, spontaneously broken phase with finite order parameter. On the other hand if $g>g_{c}$ then the coupling $g(\mu)$ flows towards increasingly strong coupling, and eventually out of reach of perturbation theory. In two dimensions the $\beta$-function has no zero and only the strong coupling phase is present.

The one-loop running of $g$ as a function of a sliding momentum scale $\mu=k$ and $\epsilon>0$ can be obtained by integrating (20),

$$
g\left(k^{2}\right)=\frac{g_{c}}{1 \pm a_{0}\left(m^{2} / k^{2}\right)^{(d-2) / 2}}
$$

with $a_{0}$ a positive constant and $m$ a mass scale; the combination $a_{0} m^{d-2}$ is just the integration constant for the differential equation. The choice of + or - sign is determined from whether one is to the left $(+)$, or to right $(-)$ of $g_{c}$, in which case $g\left(k^{2}\right)$ decreases or, respectively, increases as one flows away from the ultraviolet fixed point. It is crucial to realize that the renormalization group invariant mass scale $\sim m$ arises here as an arbitrary integration constant of the renormalization group equations, and cannot be determined from perturbative arguments alone. One can integrate the $\beta$-function equation in (20) to obtain the renormalization group invariant quantity

$$
\xi^{-1}(g)=m(g)=\text { const. } \Lambda \exp \left(-\int^{g} \frac{d g^{\prime}}{\beta\left(g^{\prime}\right)}\right)
$$

which is identified with the correlation length appearing in physical correlation functions. The multiplicative constant in front of the expression on the right hand side arises as an integration constant, and cannot be determined from perturbation theory in $g$. 
In the vicinity of the fixed point at $g_{c}$ one can do the integral in (23), using (21) and the resulting linearized expression for the $\beta$-function in the vicinity of the non-trivial ultraviolet fixed point,

$$
\beta(g) \underset{g \rightarrow g_{c}}{\sim} \beta^{\prime}\left(g_{c}\right)\left(g-g_{c}\right)+\cdots
$$

and one finds

$$
\xi^{-1}(g)=m(g) \propto \Lambda\left|g-g_{c}\right|^{v}
$$

with a correlation length exponent $v=-1 / \beta^{\prime}\left(g_{c}\right) \sim 1 /(d-2)+\cdots$. Thus the correlation length $\xi(g)$ diverges as one approaches the fixed point at $g_{c}$.

It is important to note that the above results can be tested experimentally. A recent sophisticated space shuttle experiment (Lipa et al. 2003) has succeeded in measuring the specific heat exponent $\alpha=2-3 v$ of superfluid Helium (which is supposed to share the same universality class as the $N=2$ non-linear $\sigma$-model, with the complex phase of the superfluid condensate acting as the order parameter) to very high accuracy

$$
\alpha=-0.0127(3)
$$

Previous theoretical predictions for the $N=2$ model include the most recent fourloop $4-\epsilon$ continuum result $\alpha=-0.01126(10)$, a recent lattice Monte Carlo estimate $\alpha=-0.0146(8)$, and the lattice variational renormalization group prediction $\alpha=-0.0125(39)$. One more point that should be mentioned here is that in the large $N$ limit the non-linear $\sigma$-model can be solved exactly. This allows an independent verification of the correctness of the general ideas presented earlier, as well as a direct comparison of explicit results for universal quantities. The general shape of $\beta(g)$ is of the type shown in Fig. 1, with $g_{c}$ a stable non-trivial UV fixed point, and $g=0$ and $g=\infty$ two stable (trivial) IR fixed points.

Perhaps the core message one gains from the discussion of the non-linear $\sigma$-model in $d>2$ is that:

The model provides a specific example of a theory which is not perturbatively renormalizable in the traditional sense, and for which the naive perturbative expansion in fixed dimension leads to uncontrollable divergences and inconsistent results.

Fig. 1 The $\beta$-function for the non-linear $\sigma$-model in the $2+\epsilon$ expansion and in the large- $N$ limit, for $d>2$

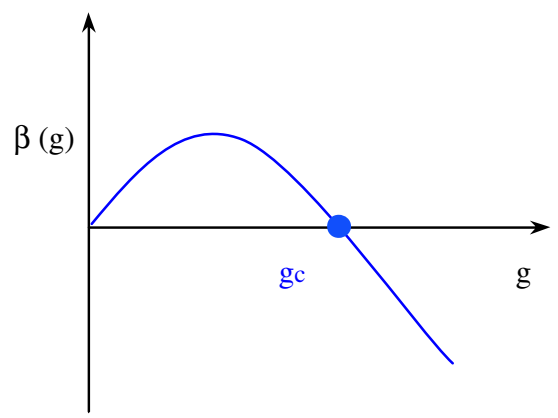


Yet the model can be constructed perturbatively in terms of a double expansion in $g$ and $\epsilon=d-2$. This new perturbative expansion, combined with the renormalization group, in the end provides explicit and detailed information about universal scaling properties of the theory in the vicinity of the non-trivial ultraviolet point at $g_{c}$.

And finally, that the continuum field theory predictions obtained this way generally agree, for distances much larger than the cutoff scale, with lattice results, and, perhaps most importantly, with high precision experiments on systems belonging to the same universality class of the $O(N)$ model. Indeed the theory results provide one of the most accurate predictions of quantum field theory to date!

\section{Phases of gravity in $2+\epsilon$ dimensions}

Can any of these lessons be applied to gravity? In two dimensions the gravitational coupling becomes dimensionless, $G \sim \Lambda^{2-d}$, and the theory appears therefore perturbatively renormalizable. In spite of the fact that the gravitational action reduces to a topological invariant in two dimensions, it would seem meaningful to try to construct, in analogy to what was suggested originally by Wilson for scalar field theories, the theory perturbatively as a double series in $\epsilon=d-2$ and $G$. One first notices though that in pure Einstein gravity, with Lagrangian density

$$
\mathcal{L}=-\frac{1}{16 \pi G_{0}} \sqrt{g} R,
$$

the bare coupling $G_{0}$ can be completely reabsorbed by a field redefinition

$$
g_{\mu \nu}=\omega g_{\mu \nu}^{\prime}
$$

with $\omega$ is a constant, and thus the renormalization properties of $G_{0}$ have no physical meaning for this theory. The situation changes though when one introduces a second dimensionful quantity to compare to. In the pure gravity case this contribution is naturally supplied by the cosmological constant term proportional to $\lambda_{0}$,

$$
\mathcal{L}=-\frac{1}{16 \pi G_{0}} \sqrt{g} R+\lambda_{0} \sqrt{g}
$$

Under a rescaling of the metric as in (28) one has

$$
\mathcal{L}=-\frac{1}{16 \pi G_{0}} \omega^{d / 2-1} \sqrt{g^{\prime}} R^{\prime}+\lambda_{0} \omega^{d / 2} \sqrt{g^{\prime}}
$$

which is interpreted as a rescaling of the two bare couplings

$$
G_{0} \rightarrow \omega^{-d / 2+1} G_{0}, \quad \lambda_{0} \rightarrow \lambda_{0} \omega^{d / 2}
$$

leaving the dimensionless combination $G_{0}^{d} \lambda_{0}^{d-2}$ unchanged. Therefore only the latter combination has physical meaning in pure gravity. In particular, one can always 
choose the scale $\omega=\lambda_{0}^{-2 / d}$ so as to adjust the volume term to have a unit coefficient. The $2+\epsilon$ expansion for pure gravity then proceeds as follows. First the gravitational part of the action

$$
\mathcal{L}=-\frac{\mu^{\epsilon}}{16 \pi G} \sqrt{g} R
$$

with $G$ dimensionless and $\mu$ an arbitrary momentum scale, is expanded by setting

$$
g_{\mu \nu} \rightarrow \bar{g}_{\mu \nu}=g_{\mu \nu}+h_{\mu \nu}
$$

where $g_{\mu \nu}$ is the classical background field and $h_{\mu \nu}$ the small quantum fluctuation. The quantity $\mathcal{L}$ in (32) is naturally identified with the bare Lagrangian, and the scale $\mu$ with a microscopic ultraviolet cutoff $\Lambda$, the inverse lattice spacing in a lattice formulation. Since the resulting perturbative expansion is generally reduced to the evaluation of Gaussian integrals, the original constraint (in the Euclidean theory)

$$
\operatorname{det} g_{\mu \nu}(x)>0
$$

is no longer enforced the same is not true in the lattice regulated theory, where it plays an important role. In order to perform the perturbative calculation of the oneloop divergences a gauge fixing term needs to be added, in the form of a background harmonic gauge condition,

$$
\mathcal{L}_{g f}=\frac{1}{2} \alpha \sqrt{g} g_{v \rho}\left(\nabla_{\mu} h^{\mu \nu}-\frac{1}{2} \beta g^{\mu \nu} \nabla_{\mu} h\right)\left(\nabla_{\lambda} h^{\lambda \rho}-\frac{1}{2} \beta g^{\lambda \rho} \nabla_{\lambda} h\right)
$$

with $h^{\mu \nu}=g^{\mu \alpha} g^{\nu \beta} h_{\alpha \beta}, h=g^{\mu \nu} h_{\mu \nu}$ and $\nabla_{\mu}$ the covariant derivative with respect to the background metric $g_{\mu \nu}$; here $\alpha$ and $\beta$ are some gauge fixing parameters. The gauge fixing term also gives rise to a Faddeev-Popov ghost contribution $\mathcal{L}_{\text {ghost }}$ containing the ghost field $\psi_{\mu}$, so that the total Lagrangian becomes $\mathcal{L}+\mathcal{L}_{g f}+\mathcal{L}_{\text {ghost }}$. After the dust settles, the one-loop radiative corrections modify the total Lagrangian to

$$
\mathcal{L} \rightarrow-\frac{\mu^{\epsilon}}{16 \pi G}\left(1-\frac{b}{\epsilon} G\right) \sqrt{g} R+\lambda_{0}\left[1-\left(\frac{a_{1}}{\epsilon}+\frac{a_{2}}{\epsilon^{2}}\right) G\right] \sqrt{g}
$$

where $a_{1}$ and $a_{2}$ are some constants. Next one can make use of the freedom to rescale the metric, by setting

$$
\left[1-\left(\frac{a_{1}}{\epsilon}+\frac{a_{2}}{\epsilon^{2}}\right) G\right] \sqrt{g}=\sqrt{g^{\prime}}
$$

which restores the original unit coefficient for the cosmological constant term. The rescaling is achieved by a suitable field redefinition

$$
g_{\mu \nu}=\left[1-\left(\frac{a_{1}}{\epsilon}+\frac{a_{2}}{\epsilon^{2}}\right) G\right]^{-2 / d} g_{\mu \nu}^{\prime}
$$


Hence the cosmological term is brought back into the standard form $\lambda_{0} \sqrt{g^{\prime}}$, and one obtains for the complete Lagrangian to first order in $G$

$$
\mathcal{L} \rightarrow-\frac{\mu^{\epsilon}}{16 \pi G}\left[1-\frac{1}{\epsilon}\left(b-\frac{1}{2} a_{2}\right) G\right] \sqrt{g^{\prime}} R^{\prime}+\lambda_{0} \sqrt{g^{\prime}}
$$

where only terms singular in $\epsilon$ have been retained. In particular one notices that only the combination $b-\frac{1}{2} a_{2}$ has physical meaning, and can in fact be shown to be gauge independent. From this last result one can finally read off the renormalization of Newton's constant

$$
\frac{1}{G} \rightarrow \frac{1}{G}\left[1-\frac{1}{\epsilon}\left(b-\frac{1}{2} a_{2}\right) G\right]
$$

The $a_{2}$ contribution cancels out the gauge-dependent part of $b$, giving for the remaining contribution $b-\frac{1}{2} a_{2}=\frac{2}{3} \cdot 19$.

In the presence of an explicit renormalization scale parameter $\mu$ the $\beta$-function for pure gravity is obtained by requiring the independence of the quantity $G_{e}$ (here identified as an effective coupling constant, with lowest order radiative corrections included) from the original renormalization scale $\mu$,

$$
\begin{aligned}
\mu \frac{d}{d \mu} G_{e} & =0 \\
\frac{1}{G_{e}} & \equiv \frac{\mu^{\epsilon}}{G(\mu)}\left[1-\frac{1}{\epsilon}\left(b-\frac{1}{2} a_{2}\right) G(\mu)\right] .
\end{aligned}
$$

To first order in $G$, one has from (41)

$$
\mu \frac{\partial}{\partial \mu} G=\beta(G)=\epsilon G-\beta_{0} G^{2}+O\left(G^{3}, G^{2} \epsilon\right)
$$

with, by explicit calculation, $\beta_{0}=\frac{2}{3} \cdot 19$. From the procedure outlined above it is clear that $G$ is the only coupling that is scale-dependent in pure gravity.

Matter fields can be included as well. When $N_{S}$ scalar fields and $N_{F}$ Majorana fermion fields are added, the results of (40) and (41) are modified to

$$
b \rightarrow b-\frac{2}{3} c
$$

with $c=N_{S}+\frac{1}{2} N_{F}$, and therefore for the combined $\beta$-function of (42) to one-loop order one has $\beta_{0}=\frac{2}{3}(19-c)$. One noteworthy aspect of the perturbative calculation is the appearance of a non-trivial ultraviolet fixed point at $G_{c}=(d-2) / \beta_{0}$ for which $\beta\left(G_{c}\right)=0$, whose physical significance is discussed below. 
Fig. 2 The renormalization group $\beta$-function for gravity in $2+\epsilon$ dimensions. The arrows indicate the coupling constant flow as one approaches increasingly larger distance scales

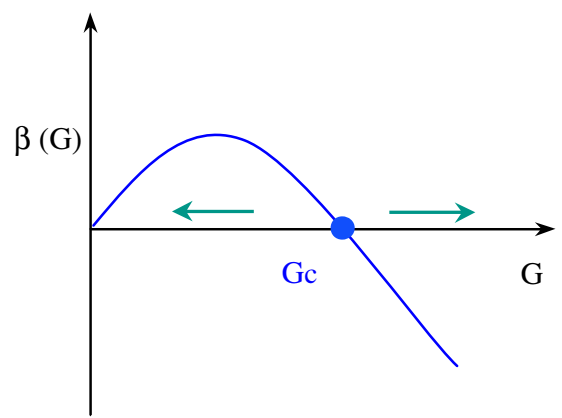

In the meantime the calculations have been laboriously extended to two loops, with the result

$$
\mu \frac{\partial}{\partial \mu} G=\beta(G)=\epsilon G-\beta_{0} G^{2}-\beta_{1} G^{3}+O\left(G^{4}, G^{3} \epsilon, G^{2} \epsilon^{2}\right)
$$

with $\beta_{0}=\frac{2}{3}(25-c)$ and $\beta_{1}=\frac{20}{3}(25-c)$. The gravitational $\beta$-function of (42) and (44) determines the scale dependence of Newton's constant $G$ for $d$ close to two. It has the general shape shown in Fig. 2. Because one is left, for the reasons described above, with a single coupling constant in the pure gravity case, the discussion becomes in fact quite similar to the non-linear $\sigma$-model case.

For a qualitative discussion of the physics it will be simpler in the following to just focus on the one loop result of (42); the inclusion of the two-loop correction does not alter the qualitative conclusions by much, as it has the same sign as the lower order, one-loop term. Depending on whether one is on the right $\left(G>G_{c}\right)$ or on the left $\left(G<G_{c}\right)$ of the non-trivial ultraviolet fixed point at

$$
G_{c}=\frac{d-2}{\beta_{0}}+O\left((d-2)^{2}\right)
$$

(with $G_{c}$ positive provided one has $c<25$ ) the coupling will either flow to increasingly larger values of $G$, or flow towards the Gaussian fixed point at $G=0$, respectively. The running of $G$ as a function of a sliding momentum scale $\mu=k$ in pure gravity can be obtained by integrating (42), and one has

$$
G\left(k^{2}\right) \simeq G_{c}\left[1+a_{0}\left(\frac{m^{2}}{k^{2}}\right)^{(d-2) / 2}+\cdots\right]
$$

with $a_{0}$ a positive constant and $m$ a mass scale. The choice of + or - sign is determined from whether one is to the left $(+)$, or to right $(-)$ of $G_{c}$, in which case the effective $G\left(k^{2}\right)$ decreases or, respectively, increases as one flows away from the ultraviolet fixed point towards lower momenta, or larger distances. Physically the two solutions represent a screening $\left(G<G_{c}\right)$ and an anti-screening $\left(G>G_{c}\right)$ situation. 
The renormalization group invariant mass scale $\sim m$ arises here as an arbitrary integration constant of the renormalization group equations. At energies sufficiently high to become comparable to the ultraviolet cutoff, the gravitational coupling $G$ flows towards the ultraviolet fixed point $G\left(k^{2}\right) \sim_{k^{2} \rightarrow \Lambda^{2}} G(\Lambda)$ where $G(\Lambda)$ is the coupling at the cutoff scale $\Lambda$, to be identified with the bare or lattice coupling. Note that the quantum correction involves a new physical, renormalization group invariant scale $\xi=1 / m$ which cannot be fixed perturbatively, and whose size determines the scale for the quantum effects. In terms of the bare coupling $G(\Lambda)$, it is given by

$$
m=A_{m} \cdot \Lambda \exp \left(-\int^{G(\Lambda)} \frac{d G^{\prime}}{\beta\left(G^{\prime}\right)}\right)
$$

which just follows from integrating $\mu \frac{\partial}{\partial \mu} G=\beta(G)$ and then setting $\mu \rightarrow \Lambda$. The constant $A_{m}$ on the r.h.s. of (47) cannot be determined perturbatively, and needs to be computed by non-perturbative (lattice) methods.

At the fixed point $G=G_{c}$ the theory is scale invariant by definition. In statistical field theory language the fixed point corresponds to a phase transition, where the correlation length $\xi=1 / m$ diverges and the theory becomes scale (conformally) invariant. In general in the vicinity of the fixed point, for which $\beta(G)=0$, one can write

$$
\beta(G) \underset{G \rightarrow G_{c}}{\sim} \beta^{\prime}\left(G_{c}\right)\left(G-G_{c}\right)+O\left(\left(G-G_{c}\right)^{2}\right)
$$

If one then defines the exponent $v$ by

$$
\beta^{\prime}\left(G_{c}\right)=-1 / v
$$

then from (47) one has by integration in the vicinity of the fixed point

$$
m \underset{G \rightarrow G_{c}}{\sim} \Lambda \cdot A_{m}\left|G(\Lambda)-G_{c}\right|^{\nu}
$$

which is why $v$ is often referred to as the mass gap exponent. Solving the above equation (with $\Lambda \rightarrow k$ ) for $G(k)$ one obtains back (46). The discussion given above is not altered significantly, at least in its qualitative aspects, by the inclusion of the two-loop correction of (44). One finds

$$
\begin{aligned}
G_{c} & =\frac{3}{2(25-c)} \epsilon-\frac{45}{2(25-c)^{2}} \epsilon^{2}+\cdots \\
v^{-1} & =\epsilon+\frac{15}{25-c} \epsilon^{2}+\cdots
\end{aligned}
$$

which gives, for pure gravity without matter $(c=0)$ in four dimensions, to lowest order $v^{-1}=2$, and $v^{-1} \approx 4.4$ at the next order. The key question raised by the perturbative calculations is therefore: what remains of the above phase transition in four 
dimensions, how are the two phases of gravity characterized there non-perturbatively, and what is the value of the exponent $v$ determining the running of $G$ in the vicinity of the fixed point in four dimensions.

\section{Lattice regularized quantum gravity}

The following section is based on the lattice discretized description of gravity originally due to Regge, where the Einstein theory is expressed in terms of a simplicial decomposition of space-time manifolds. Its use in quantum gravity is prompted by the desire to make use of techniques developed in lattice gauge theories, but with a lattice which reflects the structure of space-time rather than just providing a flat passive background. It also allows one to use powerful nonperturbative analytical techniques of statistical mechanics as well as numerical methods.

On the lattice the infinite number of degrees of freedom in the continuum is restricted, by considering Riemannian spaces described by only a finite number of variables, the geodesic distances between neighboring points. Such spaces are taken to be flat almost everywhere and referred to as piecewise linear. The elementary building blocks for $d$-dimensional space-time are simplices of dimension $d$. A 0 -simplex is a point, a 1-simplex is an edge, a 2-simplex is a triangle, a 3-simplex is a tetrahedron. A $d$-simplex is a $d$-dimensional object with $d+1$ vertices and $d(d+1) / 2$ edges connecting them. It has the important property that the values of its edge lengths specify the shape, and therefore the relative angles, uniquely. A simplicial complex can then be viewed as a set of simplices glued together in such a way that either two simplices are disjoint or they touch at a common face. The relative position of points on the lattice is thus completely specified by an incidence matrix (it tells which point is next to which) and the edge lengths, and this in turn induces a metric structure on the piecewise linear space. Finally the polyhedron constituting the union of all the simplices of dimension $d$ is called a geometrical complex or skeleton.

Consider a general simplicial lattice in $d$ dimensions, made out of a collection of flat $d$-simplices glued together at their common faces so as to constitute a triangulation of a smooth continuum manifold, such as the $d$-torus, or the surface of a sphere. If we focus on one such $d$-simplex, it will itself contain sub-simplices of smaller dimensions; as an example in four dimensions a given four-simplex will contain five tetrahedra, ten triangles (also referred to as hinges in four dimensions), ten edges and five vertices. In general, an $n$-simplex will contain $\left(\begin{array}{l}n+1 \\ k+1\end{array}\right) k$-simplices in its boundary. It will be natural in the following to label simplices by the letter $s$, faces by $f$ and hinges by $h$. A general connected, oriented simplicial manifold consisting of $N_{s} d$-simplices will also be characterized by an incidence matrix $I_{s, s^{\prime}}$, whose matrix element $I_{s, s^{\prime}}$ is chosen to be equal to one if the two simplices labeled by $s$ and $s^{\prime}$ share a common face, and zero otherwise.

The geometry of the interior of a $d$-simplex is assumed to be flat, and is therefore completely specified by the lengths of its $d(d+1) / 2$ edges. Let $x^{\mu}(i)$ be the $\mu$ th coordinate of the $i$ th site. For each pair of neighboring sites $i$ and $j$ the link length squared is given by the usual expression

$$
l_{i j}^{2}=\eta_{\mu \nu}[x(i)-x(j)]^{\mu}[x(i)-x(j)]^{v}
$$


with $\eta_{\mu \nu}$ the flat metric. It is therefore natural to associate, within a given simplex $s$, an edge vector $l_{i j}^{\mu}(s)$ with the edge connecting site $i$ to site $j$. When focusing on one such $n$-simplex it will be convenient to label the vertices by $0,1,2,3, \ldots, n$ and denote the square edge lengths by $l_{01}^{2}=l_{10}^{2}, \ldots, l_{0 n}^{2}$. The simplex can then be spanned by the set of $n$ vectors $e_{1}, \ldots, e_{n}$ connecting the vertex 0 to the other vertices. To the remaining edges within the simplex one then assigns vectors $e_{i j}=e_{i}-e_{j}$ with $1 \leq i<j \leq n$. One has therefore $n$ independent vectors, but $\frac{1}{2} n(n+1)$ invariants given by all the edge lengths squared within $s$. In the interior of a given $n$-simplex one can also assign a second, orthonormal (Lorentz) frame, which we will denote in the following by $\Sigma(s)$. The expansion coefficients relating this orthonormal frame to the one specified by the $n$ directed edges of the simplex associated with the vectors $e_{i}$ is the lattice analog of the $n$-bein or tetrad $e_{\mu}^{a}$. Within each $n$-simplex one can define a metric

$$
g_{i j}(s)=e_{i} \cdot e_{j}
$$

with $1 \leq i, j \leq n$, and which in the Euclidean case is positive definite. In components one has $g_{i j}=\eta_{a b} e_{i}^{a} e_{j}^{b}$. In terms of the edge lengths $l_{i j}=\left|e_{i}-e_{j}\right|$, the metric is given by

$$
g_{i j}(s)=\frac{1}{2}\left(l_{0 i}^{2}+l_{0 j}^{2}-l_{i j}^{2}\right) .
$$

Comparison with the standard expression for the invariant interval $d s^{2}=g_{\mu \nu} d x^{\mu} d x^{\nu}$ confirms that for the metric in (54) coordinates have been chosen along the $n e_{i}$ directions.

The volume of a general $n$-simplex is given by the $n$-dimensional generalization of the well-known formula for a tetrahedron, namely

$$
V_{n}(s)=\frac{1}{n !} \sqrt{\operatorname{det} g_{i j}(s)}
$$

It is possible to associate $p$-forms with lower dimensional objects within a simplex, which will become useful later. With each face $f$ of an $n$-simplex (in the shape of a tetrahedron in four dimensions) one can associate a vector perpendicular to the face

$$
\omega(f)_{\alpha}=\epsilon_{\alpha \beta_{1} \ldots \beta_{n-1}} e_{(1)}^{\beta_{1}} \ldots e_{(n-1)}^{\beta_{n-1}}
$$

where $e_{(1)}, \ldots, e_{(n-1)}$ are a set of oriented edges belonging to the face $f$, and $\epsilon_{\alpha_{1}, \ldots, \alpha_{n}}$ is the sign of the permutation $\left(\alpha_{1}, \ldots, \alpha_{n}\right)$.

The volume of the face $f$ is then given by

$$
V_{n-1}(f)=\left(\sum_{\alpha=1}^{n} \omega_{\alpha}^{2}(f)\right)^{1 / 2}
$$


Similarly, one can consider a hinge (a triangle in four dimensions) spanned by edges $e_{(1)}, \ldots, e_{(n-2)}$. One defines the (un-normalized) hinge bivector

$$
\omega(h)_{\alpha \beta}=\epsilon_{\alpha \beta \gamma_{1} \ldots \gamma_{n-2}} e_{(1)}^{\gamma_{1}} \ldots e_{(n-2)}^{\gamma_{n-2}}
$$

with the area of the hinge then given by

$$
V_{n-2}(h)=\frac{1}{(n-2) !}\left(\sum_{\alpha<\beta} \omega_{\alpha \beta}^{2}(h)\right)^{1 / 2}
$$

Next, in order to introduce curvature, one needs to define the dihedral angle between faces in an $n$-simplex. In an $n$-simplex $s$ two $n-1$-simplices $f$ and $f^{\prime}$ will intersect on a common $n-2$-simplex $h$, and the dihedral angle at the specified hinge $h$ is defined as

$$
\cos \theta\left(f, f^{\prime}\right)=\frac{\omega(f)_{n-1} \cdot \omega\left(f^{\prime}\right)_{n-1}}{V_{n-1}(f) V_{n-1}\left(f^{\prime}\right)}
$$

where the scalar product appearing on the r.h.s. can be re-written in terms of squared edge lengths using

$$
\omega_{n} \cdot \omega_{n}^{\prime}=\frac{1}{(n !)^{2}} \operatorname{det}\left(e_{i} \cdot e_{j}^{\prime}\right)
$$

and $e_{i} \cdot e_{j}^{\prime}$ in turn expressed in terms of squared edge lengths by the use of (54) (Note that the dihedral angle $\theta$ would have to be defined as $\pi$ minus the arccosine of the expression on the r.h.s. if the orientation for the $e$ 's had been chosen in such a way that the $\omega$ 's would all point from the face $f$ inward into the simplex $s$ ). As an example, in two dimensions and within a given triangle, two edges will intersect at a vertex, giving $\theta$ as the angle between the two edges. In three dimensions within a given simplex two triangles will intersect at a given edge, while in four dimension two tetrahedra will meet at a triangle. For the special case of an equilateral $n$-simplex, one has simply $\theta=\arccos \frac{1}{n}$.

In a piecewise linear space curvature is detected by going around elementary loops which are dual to a $(d-2)$-dimensional subspace. From the dihedral angles associated with the faces of the simplices meeting at a given hinge $h$ one can compute the deficit angle $\delta(h)$, defined as

$$
\delta(h)=2 \pi-\sum_{s \supset h} \theta(s, h)
$$

where the sum extends over all simplices $s$ meeting on $h$. It then follows that the deficit angle $\delta$ is a measure of the curvature at $h$.

Since the interior of each simplex $s$ is assumed to be flat, one can assign to it a Lorentz frame $\Sigma(s)$. Furthermore inside $s$ one can define a $d$-component vector 
$\phi(s)=\left(\phi_{0}, \ldots, \phi_{d-1}\right)$. Under a Lorentz transformation of $\Sigma(s)$, described by the $d \times d$ matrix $\Lambda(s)$ satisfying the usual relation for Lorentz transformation matrices

$$
\Lambda^{T} \eta \Lambda=\eta
$$

the vector $\phi(s)$ will rotate to

$$
\phi^{\prime}(s)=\Lambda(s) \phi(s)
$$

The base edge vectors $e_{i}^{\mu}=l_{0 i}^{\mu}(s)$ themselves are of course an example of such a vector. Next consider two $d$-simplices, individually labeled by $s$ and $s^{\prime}$, sharing a common face $f\left(s, s^{\prime}\right)$ of dimensionality $d-1$. It will be convenient to label the $d$ edges residing in the common face $f$ by indices $i, j=1, \ldots, d$. Within the first simplex $s$ one can then assign a Lorentz frame $\Sigma(s)$, and similarly within the second $s^{\prime}$ one can assign the frame $\Sigma\left(s^{\prime}\right)$. The $\frac{1}{2} d(d-1)$ edge vectors on the common interface $f\left(s, s^{\prime}\right)$ (corresponding physically to the same edges, viewed from two different coordinate systems) are expected to be related to each other by a Lorentz rotation $\mathbf{R}$,

$$
l_{i j}^{\mu}\left(s^{\prime}\right)=R_{v}^{\mu}\left(s^{\prime}, s\right) l_{i j}^{v}(s)
$$

Under individual Lorentz rotations in $s$ and $s^{\prime}$ one has of course a corresponding change in $\mathbf{R}$, namely $\mathbf{R} \rightarrow \Lambda\left(s^{\prime}\right) \mathbf{R}\left(s^{\prime}, s\right) \Lambda(s)$. In the Euclidean $d$-dimensional case $\mathbf{R}$ is an orthogonal matrix, element of the group SO $(d)$. In the absence of torsion, one can use the matrix $\mathbf{R}\left(s^{\prime}, s\right)$ to describes the parallel transport of any vector $\phi^{\mu}$ from simplex $s$ to a neighboring simplex $s^{\prime}$,

$$
\phi^{\mu}\left(s^{\prime}\right)=R_{\nu}^{\mu}\left(s^{\prime}, s\right) \phi^{v}(s)
$$

$\mathbf{R}$ therefore describes a lattice version of the connection. Indeed in the continuum such a rotation would be described by the matrix

$$
R_{v}^{\mu}=\left(e^{\Gamma \cdot d x}\right)_{v}^{\mu}
$$

with $\Gamma_{\mu \nu}^{\lambda}$ the affine connection. The coordinate increment $d x$ is interpreted as joining the center of $s$ to the center of $s^{\prime}$, thereby intersecting the face $f\left(s, s^{\prime}\right)$. On the other hand, in terms of the Lorentz frames $\Sigma(s)$ and $\Sigma\left(s^{\prime}\right)$ defined within the two adjacent simplices, the rotation matrix is given instead by

$$
R_{b}^{a}\left(s^{\prime}, s\right)=e_{\mu}^{a}\left(s^{\prime}\right) e_{b}^{v}(s) R_{\nu}^{\mu}\left(s^{\prime}, s\right)
$$

(this last matrix reduces to the identity if the two orthonormal bases $\Sigma(s)$ and $\Sigma\left(s^{\prime}\right)$ are chosen to be the same, in which case the connection is simply given by $R\left(s^{\prime}, s\right)_{\mu}{ }^{\nu}=$ $\left.e_{\mu}{ }^{a} e^{v}{ }_{a}\right)$. Note that it is possible to choose coordinates so that $\mathbf{R}\left(s, s^{\prime}\right)$ is the unit matrix for one pair of simplices, but it will not then be unity for all other pairs if curvature is present. 
This last set of results will be useful later when discussing lattice Fermions. Let us consider here briefly the problem of how to introduce lattice spin rotations. Given in $d$ dimensions the above rotation matrix $\mathbf{R}\left(s^{\prime}, s\right)$, the spin connection $\mathbf{S}\left(s, s^{\prime}\right)$ between two neighboring simplices $s$ and $s^{\prime}$ is defined as follows. Consider $\mathbf{S}$ to be an element of the $2^{v}$-dimensional representation of the covering group of $\operatorname{SO}(d), \operatorname{Spin}(d)$, with $d=2 v$ or $d=2 v+1$, and for which $S$ is a matrix of dimension $2^{v} \times 2^{v}$. Then $\mathbf{R}$ can be written in general as

$$
\mathbf{R}=\exp \left[\frac{1}{2} \sigma^{\alpha \beta} \theta_{\alpha \beta}\right]
$$

where $\theta_{\alpha \beta}$ is an antisymmetric matrix. The $\sigma$ 's are $\frac{1}{2} d(d-1) d \times d$ matrices, generators of the Lorentz group $(\mathrm{SO}(d)$ in the Euclidean case, and $\mathrm{SO}(d-1,1)$ in the Lorentzian case), whose explicit form is

$$
\left[\sigma_{\alpha \beta}\right]_{\delta}^{\gamma}=\delta_{\alpha}^{\gamma} \eta_{\beta \delta}-\delta_{\beta}^{\gamma} \eta_{\alpha \delta} \text {. }
$$

For fermions the corresponding spin rotation matrix is then obtained from

$$
\mathbf{S}=\exp \left[\frac{i}{4} \gamma^{\alpha \beta} \theta_{\alpha \beta}\right]
$$

with generators $\gamma^{\alpha \beta}=\frac{1}{2 i}\left[\gamma^{\alpha}, \gamma^{\beta}\right]$, and with the Dirac matrices $\gamma^{\alpha}$ satisfying as usual $\gamma^{\alpha} \gamma^{\beta}+\gamma^{\beta} \gamma^{\alpha}=2 \eta^{\alpha \beta}$. Taking appropriate traces, one can obtain a direct relationship between the original rotation matrix $\mathbf{R}\left(s, s^{\prime}\right)$ and the corresponding spin rotation matrix $\mathbf{S}\left(s, s^{\prime}\right)$

$$
R_{\alpha \beta}=\operatorname{tr}\left(\mathbf{S}^{\dagger} \gamma_{\alpha} \mathbf{S} \gamma_{\beta}\right) / \operatorname{tr} \mathbf{1}
$$

which determines the spin rotation matrix up to a sign.

One can consider a sequence of rotations along an arbitrary path $P\left(s_{1}, \ldots, s_{n+1}\right)$ going through simplices $s_{1}, \ldots, s_{n+1}$, whose combined rotation matrix is given by

$$
\mathbf{R}(P)=\mathbf{R}\left(s_{n+1}, s_{n}\right) \cdots \mathbf{R}\left(s_{2}, s_{1}\right)
$$

and which describes the parallel transport of an arbitrary vector from the interior of simplex $s_{1}$ to the interior of simplex $s_{n+1}$,

$$
\phi^{\mu}\left(s_{n+1}\right)=R^{\mu}{ }_{v}(P) \phi^{\nu}\left(s_{1}\right) .
$$

If the initial and final simplices $s_{n+1}$ and $s_{1}$ coincide, one obtains a closed path $C\left(s_{1}, \ldots, s_{n}\right)$, for which the associated expectation value can be considered as the gravitational analog of the Wilson loop. Its combined rotation is given by

$$
\mathbf{R}(C)=\mathbf{R}\left(s_{1}, s_{n}\right) \cdots \mathbf{R}\left(s_{2}, s_{1}\right)
$$


Fig. 3 Elementary polygonal path around a hinge (triangle) in four dimensions. The hinge $A B C$, contained in the simplex $A B C D E$, is encircled by the polygonal path $H$ connecting the surrounding vertices, which reside in the dual lattice. One such vertex is contained within the simplex $A B C D E$

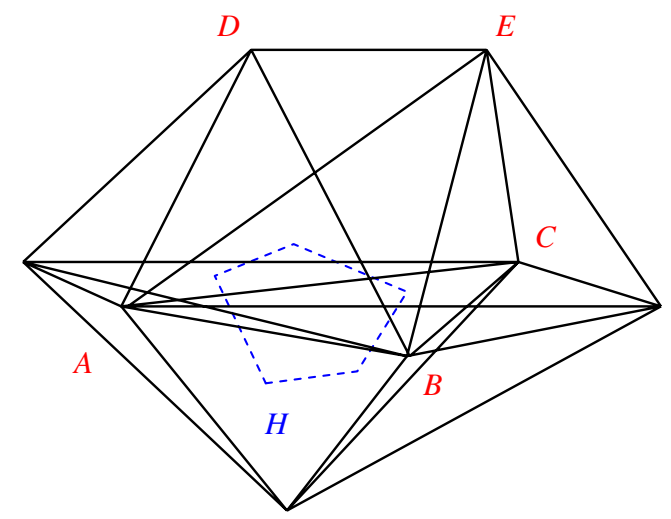

Under Lorentz transformations within each simplex $s_{i}$ along the path one has a pairwise cancellation of the $\Lambda\left(s_{i}\right)$ matrices except at the endpoints, giving in the closed loop case

$$
\mathbf{R}(C) \rightarrow \Lambda\left(s_{1}\right) \mathbf{R}(C) \Lambda^{T}\left(s_{1}\right)
$$

Clearly the deviation of the matrix $\mathbf{R}(C)$ from unity is a measure of curvature. Also, the trace $\operatorname{tr} \mathbf{R}(C)$ is independent of the choice of Lorentz frames.

Of particular interest is the elementary loop associated with the smallest non-trivial, segmented parallel transport path one can build on the lattice. One such polygonal path in four dimensions is shown in Fig. 3. In general consider a $(d-2)$-dimensional simplex (hinge) $h$, which will be shared by a certain number $m$ of $d$-simplices, sequentially labeled by $s_{1}, \ldots, s_{m}$, and whose common faces $f\left(s_{1}, s_{2}\right), \ldots, f\left(s_{m-1}, s_{m}\right)$ will also contain the hinge $h$. Thus in four dimensions several four-simplices will contain, and therefore encircle, a given triangle (hinge). In three dimensions the path will encircle an edge, while in two dimensions it will encircle a site. Thus for each hinge $h$ there is a unique elementary closed path $C_{h}$ for which one again can define the ordered product

$$
\mathbf{R}\left(C_{h}\right)=\mathbf{R}\left(s_{1}, s_{m}\right) \cdots \mathbf{R}\left(s_{2}, s_{1}\right)
$$

The hinge $h$, being geometrically an object of dimension $(d-2)$, is naturally represented by a tensor of rank $(d-2)$, referred to a coordinate system in $h$ : an edge vector $l_{h}^{\mu}$ in $d=3$, and an area bi-vector $\frac{1}{2}\left(l_{h}^{\mu} l_{h}^{\prime \nu}-l_{h}^{\nu} l_{h}^{\prime \mu}\right)$ in $d=4$, etc. Following (58) it will therefore be convenient to define a hinge bi-vector $U$ in any dimension as

$$
U_{\mu \nu}(h)=\mathcal{N} \epsilon_{\mu \nu \alpha_{1} \alpha_{d-2}} l_{(1)}^{\alpha_{1}} \ldots l_{(d-2)}^{\alpha_{d-2}},
$$

normalized, by the choice of the constant $\mathcal{N}$, in such a way that $U_{\mu \nu} U^{\mu \nu}=2$. In four dimensions

$$
U_{\mu \nu}(h)=\frac{1}{2 A_{h}} \epsilon_{\mu \nu \alpha \beta} l_{1}^{\alpha} l_{2}^{\beta}
$$


where $l_{1}(h)$ and $l_{2}(h)$ two independent edge vectors associated with the hinge $h$, and $A_{h}$ the area of the hinge.

An important aspect related to the rotation of an arbitrary vector, when parallel transported around a hinge $h$, is the fact that, due to the hinge's intrinsic orientation, only components of the vector in the plane perpendicular to the hinge are affected. Since the direction of the hinge $h$ is specified locally by the bivector $U_{\mu \nu}$ of (79), one can write for the loop rotation matrix $\mathbf{R}$

$$
R_{\nu}^{\mu}(C)=\left(e^{\delta U}\right)^{\mu}
$$

where $C$ is now the small polygonal loop entangling the hinge $h$, and $\delta$ the deficit angle at $h$, previously defined in (62). One particularly noteworthy aspect of this last result is the fact that the area of the loop $C$ does not enter in the expression for the rotation matrix, only the deficit angle and the hinge direction.

At the same time, in the continuum a vector $V$ carried around an infinitesimal loop of area $A_{C}$ will change by

$$
\Delta V^{\mu}=\frac{1}{2} R_{\nu \lambda \sigma}^{\mu} A^{\lambda \sigma} V^{v}
$$

where $A^{\lambda \sigma}$ is an area bivector in the plane of $C$, with squared magnitude $A_{\lambda \sigma} A^{\lambda \sigma}=$ $2 A_{C}^{2}$. Since the change in the vector $V$ is given by $\delta V^{\alpha}=(\mathbf{R}-\mathbf{1})^{\alpha}{ }_{\beta} V^{\beta}$ one is led to the identification

$$
\frac{1}{2} R_{\beta \mu \nu}^{\alpha} A^{\mu \nu}=(\mathbf{R}-\mathbf{1})_{\beta}^{\alpha} .
$$

Thus the above change in $V$ can equivalently be re-written in terms of the infinitesimal rotation matrix

$$
R_{\nu}^{\mu}(C)=\left(e^{\frac{1}{2} R \cdot A}\right)_{\nu}^{\mu}
$$

where the Riemann tensor appearing in the exponent on the r.h.s. should not be confused with the rotation matrix $\mathbf{R}$ on the 1.h.s.

It is then immediate to see that the two expressions for the rotation matrix $\mathbf{R}$ in (80) and (83) will be compatible provided one uses for the Riemann tensor at a hinge $h$ the expression

$$
R_{\mu \nu \lambda \sigma}(h)=\frac{\delta(h)}{A_{C}(h)} U_{\mu \nu}(h) U_{\lambda \sigma}(h)
$$

expected to be valid in the limit of small curvatures, with $A_{C}(h)$ the area of the loop entangling the hinge $h$. Here use has been made of the geometric relationship $U_{\mu \nu} A^{\mu \nu}=2 A_{C}$. Note that the bivector $U$ has been defined to be perpendicular to 
the $(d-2)$ edge vectors spanning the hinge $h$, and lies therefore in the same plane as the loop $C$. The area $A_{C}$ is most suitably defined by introducing the notion of a dual lattice, i.e., a lattice constructed by assigning centers to the simplices, with the polygonal curve $C$ connecting these centers sequentially, and then assigning an area to the interior of this curve. One possible way of assigning such centers is by introducing perpendicular bisectors to the faces of a simplex, and locate the vertices of the dual lattice at their common intersection, a construction originally discussed by Voronoi.

The first step in writing down an invariant lattice action, analogous to the continuum Einstein-Hilbert action, is to find the lattice analog of the Ricci scalar. From the expression for the Riemann tensor at a hinge given in (84) one obtains by contraction

$$
R(h)=2 \frac{\delta(h)}{A_{C}(h)}
$$

The continuum expression $\sqrt{g} R$ is then obtained by multiplication with the volume element $V(h)$ associated with a hinge. The latter is defined by first joining the vertices of the polyhedron $C$, whose vertices lie in the dual lattice, with the vertices of the hinge $h$, and then computing its volume.

By defining the polygonal area $A_{C}$ as $A_{C}(h)=d V(h) / V^{(d-2)}(h)$, where $V^{(d-2)}(h)$ is the volume of the hinge (an area in four dimensions), one finally obtains for the Euclidean lattice action for pure gravity

$$
I_{R}\left(l^{2}\right)=-k \sum_{\text {hinges } h} \delta(h) V^{(d-2)}(h),
$$

with the constant $k=1 /(8 \pi G)$. One would have obtained the same result for the single-hinge contribution to the lattice action if one had contracted the infinitesimal form of the rotation matrix $R(h)$ in (80) with the hinge bivector $\omega_{\alpha \beta}$ of (58) (or equivalently with the bivector $U_{\alpha \beta}$ of (79) which differs from $\omega_{\alpha \beta}$ by a constant). The fact that the lattice action only involves the content of the hinge $V^{(d-2)}(h)$ (the area of a triangle in four dimensions) is quite natural in view of the fact that the rotation matrix at a hinge in (80) only involves the deficit angle, and not the polygonal area $A_{C}(h)$.

Other terms need to be added to the lattice action. Consider for example a cosmological constant term, which in the continuum theory takes the form $\lambda_{0} \int d^{d} x \sqrt{g}$. The expression for the cosmological constant term on the lattice involves the total volume of the simplicial complex. This may be written as

$$
V_{\text {total }}=\sum_{\text {simplices } s} V_{s}
$$

or equivalently as

$$
V_{\text {total }}=\sum_{\text {hinges } h} V_{h}
$$


Fig. 4 On a random simplicial lattice there are in general no preferred directions. The lattice can be deformed locally from one configuration of edges to another which has the same localized curvature, and illustrates the lattice analog of the continuum diffeomorphism invariance

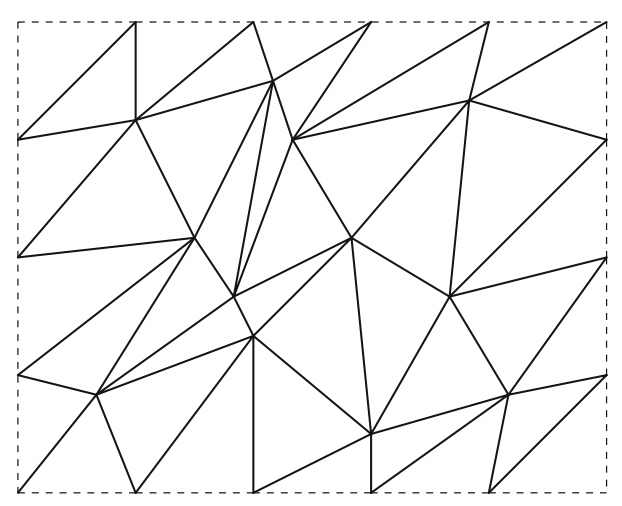

where $V_{h}$ is the volume associated with each hinge via the construction of a dual lattice, as described above. Thus one may regard the local volume element $\sqrt{g} d^{d} x$ as being represented by either $V_{h}$ (centered on $h$ ) or $V_{s}$ (centered on $s$ ).

The Regge and cosmological constant term then lead to the combined action

$$
I_{\text {latt }}\left(l^{2}\right)=\lambda_{0} \sum_{\text {simplices } s} V_{s}^{(d)}-k \sum_{\text {hinges } h} \delta_{h} V_{h}^{(d-2)}
$$

Another interesting aspect is the exact local gauge invariance of the lattice action. Consider the two-dimensional flat skeleton shown in Fig. 4. It is clear that one can move around a point on the surface, keeping all the neighbors fixed, without violating the triangle inequalities and leave all curvature invariants unchanged.

In $d$ dimensions this transformation has $d$ parameters and is an exact invariance of the action. When space is slightly curved, the invariance is in general only an approximate one, even though for piecewise linear spaces piecewise diffeomorphisms can still be defined as the set of local motions of points that leave the local contribution to the action, the measure and the lattice analogs of the continuum curvature invariants unchanged. Note that in general the gauge deformations of the edges are still constrained by the triangle inequalities. The general situation is illustrated in Fig. 4. In the limit when the number of edges becomes very large one expects the full continuum diffeomorphism group to be recovered. In general the structure of lattice local gauge transformations is rather complicated and will not be given here. These are defined as transformations acting locally on a given set of edges which leave the local lattice curvature invariant. The simplest context in which this local invariance can be exhibited explicitly is the lattice weak field expansion. From the transformation properties of the edge lengths it is clear that their transformation properties are related to those of the local metric, as already suggested for example by the identification of (54) and (90). In the quantum theory, a local gauge invariance implies the existence of conservation laws and Ward identities for $n$-point functions. 


\section{Lattice regularized path integral}

As the edge lengths $l_{i j}$ play the role of the continuum metric $g_{\mu v}(x)$, one would expect the discrete measure to involve an integration over the squared edge lengths. Indeed the induced metric at a simplex is related to the squared edge lengths within that simplex, via the expression for the invariant line element $d s^{2}=g_{\mu \nu} d x^{\mu} d x^{\nu}$. After choosing coordinates along the edges emanating from a vertex, the relation between metric perturbations and squared edge length variations for a given simplex based at 0 in $d$ dimensions is

$$
\delta g_{i j}\left(l^{2}\right)=\frac{1}{2}\left(\delta l_{0 i}^{2}+\delta l_{0 j}^{2}-\delta l_{i j}^{2}\right)
$$

For one $d$-dimensional simplex labeled by $s$ the integration over the metric is thus equivalent to an integration over the edge lengths, and one has the identity

$$
\left(\frac{1}{d !} \sqrt{\operatorname{det} g_{i j}(s)}\right)^{\sigma} \prod_{i \geq j} d g_{i j}(s)=\left(-\frac{1}{2}\right)^{\frac{d(d-1)}{2}}\left[V_{d}\left(l^{2}\right)\right]^{\sigma} \prod_{k=1}^{d(d+1) / 2} d l_{k}^{2}
$$

There are $d(d+1) / 2$ edges for each simplex, just as there are $d(d+1) / 2$ independent components for the metric tensor in $d$ dimensions. Here one is ignoring temporarily the triangle inequality constraints, which will further require all sub-determinants of $g_{i j}$ to be positive, including the obvious restriction $l_{k}^{2}>0$.

Let us discuss here briefly the simplicial inequalities which need to be imposed on the edge lengths. These are conditions on the edge lengths $l_{i j}$ such that the sites $i$ can be considered the vertices of a $d$-simplex embedded in flat $d$-dimensional Euclidean space. In one dimension, $d=1$, one requires trivially for all edge lengths $l_{i j}^{2}>0$. In higher dimensions one requires that all triangle inequalities and their higher dimensional analogs to be satisfied,

$$
\begin{aligned}
l_{i j}^{2} & >0 \\
V_{k}^{2} & =\left(\frac{1}{k !}\right)^{2} \operatorname{det} g_{i j}^{(k)}(s)>0
\end{aligned}
$$

with $k=2, \ldots, d$ for every possible choice of sub-simplex (and therefore sub-determinant) within the original simplex $s$. The extension of the measure to many simplices glued together at their common faces is then immediate. For this purpose one first needs to identify edges $l_{k}(s)$ and $l_{k^{\prime}}\left(s^{\prime}\right)$ which are shared between simplices $s$ and $s^{\prime}$,

$$
\int_{0}^{\infty} d l_{k}^{2}(s) \int_{0}^{\infty} d l_{k^{\prime}}^{2}\left(s^{\prime}\right) \delta\left[l_{k}^{2}(s)-l_{k^{\prime}}^{2}\left(s^{\prime}\right)\right]=\int_{0}^{\infty} d l_{k}^{2}(s)
$$


After summing over all simplices one derives, up to an irrelevant numerical constant, the unique functional measure for simplicial geometries

$$
\int\left[d l^{2}\right]=\int_{\epsilon}^{\infty} \prod_{s}\left[V_{d}(s)\right]^{\sigma} \prod_{i j} d l_{i j}^{2} \Theta\left[l_{i j}^{2}\right] .
$$

Here $\Theta\left[l_{i j}^{2}\right]$ is a (step) function of the edge lengths, with the property that it is equal to one whenever the triangle inequalities and their higher dimensional analogs are satisfied, and zero otherwise. The quantity $\epsilon$ has been introduced as a cutoff at small edge lengths. If the measure is non-singular for small edges, one can safely take the limit $\epsilon \rightarrow 0$. In four dimensions the lattice analog of the DeWitt measure $(\sigma=0)$ takes on a particularly simple form, namely

$$
\int\left[d l^{2}\right]=\int_{0}^{\infty} \prod_{i j} d l_{i j}^{2} \Theta\left[l_{i j}^{2}\right] .
$$

Lattice measures over the space of squared edge lengths have been used extensively in numerical simulations of simplicial quantum gravity. The derivation of the above lattice measure closely parallels the analogous procedure in the continuum.

The lattice action of (89) for pure four-dimensional Euclidean gravity then contains a cosmological constant and Regge scalar curvature term, as well as possibly higher derivative terms. It only couples edges which belong either to the same simplex or to a set of neighboring simplices, and can therefore be considered as local, just like the continuum action. It leads to a regularized lattice functional integral

$$
Z_{\text {latt }}=\int\left[d l^{2}\right] e^{-\lambda_{0} \sum_{h} V_{h}+k \sum_{h} \delta_{h} A_{h}},
$$

where, as customary, the lattice ultraviolet cutoff is set equal to one (i.e., all length scales are measured in units of the lattice cutoff). The lattice partition function $Z_{\text {latt }}$ should then be compared to the continuum Euclidean Feynman path integral of (14),

$$
Z_{\mathrm{cont}}=\int\left[d g_{\mu \nu}\right] e^{-\lambda_{0} \int d x \sqrt{g}+\frac{1}{16 \pi G} \int d x \sqrt{g} R} .
$$

Occasionally it can be convenient to include the $\lambda_{0}$-term in the measure. For this purpose one defines

$$
d \mu\left(l^{2}\right) \equiv\left[d l^{2}\right] e^{-\lambda_{0} \sum_{h} V_{h}}
$$

It should be clear that this last expression represents a fairly non-trivial quantity, both in view of the relative complexity of the expression for the volume of a simplex, and because of the generalized triangle inequality constraints already implicit in $\left[d l^{2}\right]$. But, like the continuum functional measure, it is certainly local, to the extent that each 
edge length appears only in the expression for the volume of those simplices which explicitly contain it. Furthermore, $\lambda_{0}$ sets the overall scale and can therefore be set equal to one without any loss of generality.

\section{Matter fields}

In the previous section we have discussed the construction and the invariance properties of a lattice action for pure gravity. Next a scalar field can be introduced as the simplest type of dynamical matter that can be coupled invariantly to gravity. In the continuum the scalar action for a single component field $\phi(x)$ is usually written as

$$
I[g, \phi]=\frac{1}{2} \int d x \sqrt{g}\left[g^{\mu \nu} \partial_{\mu} \phi \partial_{\nu} \phi+\left(m^{2}+\xi R\right) \phi^{2}\right]+\cdots
$$

where the dots denote scalar self-interaction terms. One way to proceed is to introduce a lattice scalar $\phi_{i}$ defined at the vertices of the simplices. The corresponding lattice action can then be obtained through a procedure by which the original continuum metric is replaced by the induced lattice metric, with the latter written in terms of squared edge lengths as in (54). Thus in two dimensions to construct a lattice action for the scalar field, one performs the replacement

$$
\begin{aligned}
g_{\mu \nu}(x) & \longrightarrow g_{i j}(\Delta) \\
\operatorname{det} g_{\mu \nu}(x) & \longrightarrow \operatorname{det} g_{i j}(\Delta) \\
g^{\mu \nu}(x) & \longrightarrow g^{i j}(\Delta) \\
\partial_{\mu} \phi \partial_{\nu} \phi & \longrightarrow \Delta_{i} \phi \Delta_{j} \phi,
\end{aligned}
$$

with the following definitions

$$
\begin{aligned}
g_{i j}(\Delta) & =\left(\begin{array}{cc}
l_{3}^{2} & \frac{1}{2}\left(-l_{1}^{2}+l_{2}^{2}+l_{3}^{2}\right) \\
\frac{1}{2}\left(-l_{1}^{2}+l_{2}^{2}+l_{3}^{2}\right) & l_{2}^{2}
\end{array}\right), \\
\operatorname{det} g_{i j}(\Delta) & =\frac{1}{4}\left[2\left(l_{1}^{2} l_{2}^{2}+l_{2}^{2} l_{3}^{2}+l_{3}^{2} l_{1}^{2}\right)-l_{1}^{4}-l_{2}^{4}-l_{3}^{4}\right] \equiv 4 A_{\Delta}^{2}, \\
g^{i j}(\Delta) & =\frac{1}{\operatorname{det} g(\Delta)}\left(\begin{array}{cc}
l_{2}^{2} & \frac{1}{2}\left(l_{1}^{2}-l_{2}^{2}-l_{3}^{2}\right) \\
\frac{1}{2}\left(l_{1}^{2}-l_{2}^{2}-l_{3}^{2}\right) & l_{3}^{2}
\end{array}\right) .
\end{aligned}
$$

The scalar field derivatives get replaced as usual by finite differences

$$
\partial_{\mu} \phi \longrightarrow\left(\Delta_{\mu} \phi\right)_{i}=\phi_{i+\mu}-\phi_{i}
$$


where the index $\mu$ labels the possible directions in which one can move away from a vertex within a given triangle. After some suitable re-arrangements one finds for the lattice action describing a massless scalar field

$$
I\left(l^{2}, \phi\right)=\frac{1}{2} \sum_{\langle i j\rangle} A_{i j}\left(\frac{\phi_{i}-\phi_{j}}{l_{i j}}\right)^{2} .
$$

Here $A_{i j}$ is the dual (Voronoi) area associated with the edge $i j$, and the symbol $\langle i j\rangle$ denotes a sum over nearest neighbor lattice vertices. It is immediate to generalize the action of (105) to higher dimensions, with the two-dimensional Voronoi volumes replaced by their higher dimensional analogs, leading to

$$
I\left(l^{2}, \phi\right)=\frac{1}{2} \sum_{\langle i j\rangle} V_{i j}^{(d)}\left(\frac{\phi_{i}-\phi_{j}}{l_{i j}}\right)^{2} .
$$

Here $V_{i j}^{(d)}$ is the dual (Voronoi) volume associated with the edge $i j$, and the sum is over all links on the lattice.

Spinor fields $\psi_{s}$ and $\bar{\psi}_{s}$ are most naturally placed at the center of each $d$-simplex $s$. As in the continuum, the construction of a suitable lattice action requires the introduction of the Lorentz group and its associated tetrad fields $e_{\mu}^{a}(s)$ within each simplex labeled by $s$. Within each simplex one can choose a representation of the Dirac gamma matrices, denoted here by $\gamma^{\mu}(s)$, such that in the local coordinate basis

$$
\left\{\gamma^{\mu}(s), \gamma^{\nu}(s)\right\}=2 g^{\mu \nu}(s)
$$

These in turn are related to the ordinary Dirac gamma matrices $\gamma^{a}$, which obey $\left\{\gamma^{a}, \gamma^{b}\right\}=2 \eta^{a b}$, by $\gamma^{\mu}(s)=e_{a}^{\mu}(s) \gamma^{a}$. so that within each simplex the tetrads $e_{\mu}^{a}(s)$ satisfy the usual relation

$$
e_{a}^{\mu}(s) e_{b}^{v}(s) \eta^{a b}=g^{\mu v}(s)
$$

In general the tetrads are not fixed uniquely within a simplex, being invariant under the local Lorentz transformations discussed earlier. In the continuum the action for a massless spinor field is given by

$$
I=\int d x \sqrt{g} \bar{\psi}(x) \gamma^{\mu} D_{\mu} \psi(x)
$$

where $D_{\mu}=\partial_{\mu}+\frac{1}{2} \omega_{\mu a b} \sigma^{a b}$ is the spinorial covariant derivative containing the spin connection $\omega_{\mu a b}$. On the lattice one then needs a rotation matrix relating the vierbeins $e_{a}^{\mu}\left(s_{1}\right)$ and $e_{a}^{\mu}\left(s_{2}\right)$ in two neighboring simplices. The matrix $\mathbf{R}\left(s_{2}, s_{1}\right)$ is such that

$$
e_{a}^{\mu}\left(s_{2}\right)=R_{v}^{\mu}\left(s_{2}, s_{1}\right) e_{a}^{v}\left(s_{1}\right)
$$


and whose spinorial representation $\mathbf{S}$ was given previously in (72). The invariant lattice action for a massless spinor then takes the simple form

$$
I=\frac{1}{2} \sum_{\text {faces } f\left(s, s^{\prime}\right)} V\left(f\left(s, s^{\prime}\right)\right) \bar{\psi}_{s} \mathbf{S}\left(\mathbf{R}\left(s, s^{\prime}\right)\right) \gamma^{\mu}\left(s^{\prime}\right) n_{\mu}\left(s, s^{\prime}\right) \psi_{s^{\prime}}
$$

where the sum extends over all interfaces $f\left(s, s^{\prime}\right)$ connecting one simplex $s$ to a neighboring simplex $s^{\prime}$. The above spinorial action can be considered analogous to the lattice Fermion action proposed originally by Wilson for non-Abelian gauge theories.

For gauge fields a locally gauge invariant action for an $\mathrm{SU}(N)$ gauge field coupled to gravity is

$$
I_{\text {gauge }}=-\frac{1}{4 g^{2}} \int d^{4} x \sqrt{g} g^{\mu \lambda} g^{\nu \sigma} F_{\mu \nu}^{a} F_{\lambda \sigma}^{a}
$$

with $F_{\mu \nu}^{a}=\nabla_{\mu} A_{v}^{a}-\nabla_{\nu} A_{\mu}^{a}+g f^{a b c} A_{\mu}^{b} A_{\nu}^{c}$ and $a=1, \ldots, N^{2}-1$. On the lattice one can follow a procedure analogous to Wilson's construction on a hypercubic lattice, with the main difference that the lattice is now simplicial. Given a link $i j$ on the lattice one assigns group element $U_{i j}$, with each $U$ an $N \times N$ unitary matrix with determinant equal to one, and such that $U_{j i}=U_{i j}^{-1}$. Then with each triangle (plaquettes) $\Delta$ labeled by the three vertices $i j k$ one associates a product of three $U$ matrices $U_{\Delta} \equiv U_{i j k}=U_{i j} U_{j k} U_{k i}$. The discrete action is then given by

$$
I_{\text {gauge }}=-\frac{1}{g^{2}} \sum_{\Delta} V_{\Delta} \frac{c}{A_{\Delta}^{2}} \operatorname{Re}\left[\operatorname{tr}\left(1-U_{\Delta}\right)\right]
$$

with 1 the unit matrix, $V_{\Delta}$ the 4-volume associated with the plaquettes $\Delta, A_{\Delta}$ the area of the triangle (plaquettes) $\Delta$, and $c$ a numerical constant of order one. One important property of the gauge lattice action of (113) is its local invariance under gauge rotations $g_{i}$ defined at the lattice vertices, and for which $U_{i j}$ on the link $i j$ transforms as

$$
U_{i j} \rightarrow g_{i} U_{i j} g_{j}^{-1}
$$

Finally one can consider a spin-3/2 field. Of course supergravity in four dimensions naturally contains a spin-3/2 gravitino, the supersymmetric partner of the graviton. In the case of $\mathcal{N}=1$ supergravity these are the only two degrees of freedom present. Consider here a spin-3/2 Majorana fermion in four dimensions, which correspond to self-conjugate Dirac spinors $\psi_{\mu}$, where the Lorentz index $\mu=1, \ldots, 4$. In flat space the action for such a field is given by the Rarita-Schwinger term

$$
\mathcal{L}_{R S}=-\frac{1}{2} \epsilon^{\alpha \beta \gamma \delta} \psi_{\alpha}^{T} C \gamma_{5} \gamma_{\beta} \partial_{\gamma} \psi_{\delta}
$$

Locally the action is invariant under the gauge transformation $\psi_{\mu}(x) \rightarrow \psi_{\mu}(x)+$ $\partial_{\mu} \epsilon(x)$, where $\epsilon(x)$ is an arbitrary local Majorana spinor. The construction of a 
suitable lattice action for the spin-3/2 particle proceeds in a way that is rather similar to what one does in the spin-1/2 case. On a simplicial manifold the Rarita-Schwinger spinor fields $\psi_{\mu}(s)$ and $\bar{\psi}_{\mu}(s)$ are most naturally placed at the center of each $d$-simplex $s$. Like the spin-1/2 case, the construction of a suitable lattice action requires the introduction of the Lorentz group and its associated vierbein fields $e_{\mu}^{a}(s)$ within each simplex labeled by $s$. Again as in the spinor case vierbeins $e_{a}^{\mu}\left(s_{1}\right)$ and $e_{a}^{\mu}\left(s_{2}\right)$ in two neighboring simplices will be related by a matrix $\mathbf{R}\left(s_{2}, s_{1}\right)$ such that

$$
e_{a}^{\mu}\left(s_{2}\right)=R_{v}^{\mu}\left(s_{2}, s_{1}\right) e_{a}^{\nu}\left(s_{1}\right)
$$

and whose spinorial representation $\mathbf{S}$ was given previously in (72). But the new ingredient in the spin-3/2 case is that, besides requiring a spin rotation matrix $\mathbf{S}\left(s_{2}, s_{1}\right)$, now one also needs the matrix $R_{\mu}^{v}\left(s, s^{\prime}\right)$ describing the corresponding parallel transport of the Lorentz vector $\psi_{\mu}(s)$ from a simplex $s_{1}$ to the neighboring simplex $s_{2}$. An invariant lattice action for a massless spin-3/2 particle takes therefore the form

$$
I=-\frac{1}{2} \sum_{\text {faces } f\left(s, s^{\prime}\right)} V\left(f\left(s, s^{\prime}\right)\right) \epsilon^{\mu \nu \lambda \sigma} \bar{\psi}_{\mu}(s) \mathbf{S}\left(\mathbf{R}\left(s, s^{\prime}\right)\right) \gamma_{\nu}\left(s^{\prime}\right) n_{\lambda}\left(s, s^{\prime}\right) R_{\sigma}^{\rho}\left(s, s^{\prime}\right) \psi_{\rho}\left(s^{\prime}\right)
$$

with

$$
\bar{\psi}_{\mu}(s) \mathbf{S}\left(\mathbf{R}\left(s, s^{\prime}\right)\right) \gamma_{\nu}\left(s^{\prime}\right) \psi_{\rho}\left(s^{\prime}\right) \equiv \bar{\psi}_{\mu \alpha}(s) S_{\beta}^{\alpha}\left(\mathbf{R}\left(s, s^{\prime}\right)\right) \gamma_{\nu}{ }^{\beta} \gamma\left(s^{\prime}\right) \psi_{\rho}^{\gamma}\left(s^{\prime}\right)
$$

and the sum $\sum_{\text {faces } \mathrm{f}\left(\mathrm{ss}^{\prime}\right)}$ extends over all interfaces $f\left(s, s^{\prime}\right)$ connecting one simplex $s$ to a neighboring simplex $s^{\prime}$. When compared to the spin-1/2 case, the most important modification is the second rotation matrix $R_{\mu}^{v}\left(s, s^{\prime}\right)$, which describes the parallel transport of the fermionic vector $\psi_{\mu}$ from the site $s$ to the site $s^{\prime}$, which is required in order to obtain locally a Lorentz scalar contribution to the action.

\section{Alternate discrete formulations}

The simplicial lattice formulation offers a natural way of representing gravitational degrees in a discrete framework by employing inherently geometric concepts such as areas, volumes and angles. It is possible though to formulate quantum gravity on a flat hypercubic lattice, in analogy to Wilson's discrete formulation for gauge theories, by putting the connection center stage. In this new set of theories the natural variables are then lattice versions of the spin connection and the vierbein. Also, because the spin connection variables appear from the very beginning, it is much easier to incorporate fermions later. Some lattice models have been based on the pure Einstein theory while others attempt to incorporate higher derivative terms.

Difficult arise when attempting to put quantum gravity on a flat hypercubic lattice a la Wilson, since it is not entirely clear what the gravity analog of the Yang-Mills connection is. In continuum formulations invariant under the Poincaré or de Sitter group 
the action is invariant under a local extension of the Lorentz transformations, but not under local translations. Local translations are replaced by diffeomorphisms which have a different nature. One set of lattice discretizations starts from the action whose local invariance group is the de Sitter group Spin(4), the covering group of SO(4). In one lattice formulation the lattice variables are gauge potentials $e_{a \mu}(n)$ and $\omega_{\mu a b}(n)$ defined on lattice sites $n$, generating local Spin(4) matrix transformations with the aid of the de Sitter generators $P_{a}$ and $M_{a b}$. The resulting lattice action reduces classically to the Einstein action with cosmological term in first order form in the limit of the lattice spacing $a \rightarrow 0$; to demonstrate the quantum equivalence one needs an additional zero torsion constraint. In the end the issue of lattice diffeomorphism invariance remains somewhat open, with the hope that such an invariance will be restored in the full quantum theory.

As an example, we will discuss here the approach of Mannion and Taylor, which relies on a four-dimensional lattice discretization of the Einstein-Cartan theory with gauge group $\operatorname{SL}(2, C)$, and does not initially require the presence of a cosmological constant, as would be the case if one had started out with the de Sitter group Spin(4). On a lattice of spacing $a$ with vertices labelled by $n$ and directions by $\mu$ one relates the relative orientations of nearest-neighbor local $\operatorname{SL}(2, C)$ frames by

$$
U_{\mu}(n)=\left[U_{-\mu}(n+\mu)\right]^{-1}=\exp \left[i B_{\mu}(n)\right]
$$

with $B_{\mu}=\frac{1}{2} a B_{\mu}^{a b}(n) J_{b a}, J_{b a}$ being the set of six generators of $\operatorname{SL}(2, C)$, the covering group of the Lorentz group $\operatorname{SO}(3,1)$, usually taken to be $\sigma_{a b}=\frac{1}{2 i}\left[\gamma_{a}, \gamma_{b}\right]$ with $\gamma_{a}$ 's the Dirac gamma matrices. The local lattice curvature is then obtained in the usual way by computing the product of four parallel transport matrices around an elementary lattice square,

$$
U_{\mu}(n) U_{v}(n+\mu) U_{-\mu}(n+\mu+v) U_{-v}(n+v)
$$

giving in the limit of small $a$ by the Baker-Hausdorff formula the value $\exp \left[i_{a} R_{\mu \nu}(n)\right]$, where $R_{\mu \nu}$ is the Riemann tensor defined in terms of the spin connection $B_{\mu}$

$$
R_{\mu \nu}=\partial_{\mu} B_{\nu}-\partial_{\nu} B_{\mu}+i\left[B_{\mu}, B_{\nu}\right]
$$

If one were to write for the action the usual Wilson lattice gauge form

$$
\sum_{n, \mu, v} \operatorname{tr}\left[U_{\mu}(n) U_{v}(n+\mu) U_{-\mu}(n+\mu+v) U_{-v}(n+v)\right]
$$

then one would obtain a curvature squared action proportional to $\sim \int R_{\mu \nu}^{a b} R_{a b}^{\mu v}$ instead of the Einstein-Hilbert one. One needs therefore to introduce lattice vierbeins $e_{\mu}{ }^{b}(n)$ on the sites by defining the matrices $E_{\mu}(n)=a e_{\mu}{ }^{a} \gamma_{a}$. Then a suitable lattice action 
is given by

$$
I=\frac{i}{16 \kappa^{2}} \sum_{n, \mu, v, \lambda, \sigma} \operatorname{tr}\left[\gamma_{5} U_{\mu}(n) U_{\nu}(n+\mu) U_{-\mu}(n+\mu+v) U_{-v}(n+v) E_{\sigma}(n) E_{\lambda}(n)\right]
$$

The latter is invariant under local $\operatorname{SL}(2, C)$ transformations $\Lambda(n)$ defined on the lattice vertices

$$
U_{\mu} \rightarrow \Lambda(n) U_{\mu}(n) \Lambda^{-1}(n+\mu)
$$

for which the curvature transforms as

$$
\begin{aligned}
& U_{\mu}(n) U_{v}(n+\mu) U_{-\mu}(n+\mu+v) U_{-v}(n+v) \\
& \quad \rightarrow \Lambda(n) U_{\mu}(n) U_{v}(n+\mu) U_{-\mu}(n+\mu+v) U_{-v}(n+v) \Lambda^{-1}(n)
\end{aligned}
$$

and the vierbein matrices as

$$
E_{\mu}(n) \rightarrow \Lambda(n) E_{\mu}(n) \Lambda^{-1}(n)
$$

Since $\Lambda(n)$ commutes with $\gamma_{5}$, the expression in (123) is invariant. The metric is then obtained as usual by

$$
g_{\mu \nu}(n)=\frac{1}{4} \operatorname{tr}\left[E_{\mu}(n) E_{\nu}(n)\right]
$$

From the expression for the lattice curvature $R_{\mu \nu}^{a b}$ given above if follows immediately that the lattice action in the continuum limit becomes

$$
I=\frac{a^{4}}{4 \kappa^{2}} \sum_{n} \epsilon^{\mu \nu \lambda \sigma} \epsilon_{a b c d} R_{\mu \nu}^{a b}(n) e_{\lambda}^{c}(n) e_{\sigma}^{d}(n)+O\left(a^{6}\right)
$$

which is the Einstein action in Cartan form

$$
I=\frac{1}{4 \kappa^{2}} \int d^{4} x \epsilon^{\mu \nu \lambda \sigma} \epsilon_{a b c d} R_{\mu \nu}^{a b} e_{\lambda}^{c} e_{\sigma}{ }^{d}
$$

with the parameter $\kappa$ identified with the Planck length. One can add more terms to the action; in this theory a cosmological term can be represented by

$$
\lambda_{0} \sum_{n} \epsilon^{\mu \nu \lambda \sigma} \operatorname{tr}\left[\gamma_{5} E_{\mu}(n) E_{v}(n) E_{\sigma}(n) E_{\lambda}(n)\right]
$$


Both (123) and (130) are locally $\operatorname{SL}(2, C)$ invariant. The functional integral is then given by

$$
Z=\int \prod_{n, \mu} d B_{\mu}(n) \prod_{n, \sigma} d E_{\sigma}(n) \exp \{-I(B, E)\}
$$

and from it one can then compute suitable quantum averages. Here $d B_{\mu}(n)$ is the Haar measure for $\operatorname{SL}(2, C)$; it is less clear how to choose the integration measure over the $E_{\sigma}$ 's, and how it should suitably constrained, which obscures the issue of diffeomorphism invariance in this theory.

There is another way of discretizing gravity, still using largely geometric concepts as is done in the Regge theory. In the dynamical triangulation approach due to David one fixes the edge lengths to unity, and varies the incidence matrix. As a result the volume of each simplex is fixed at

$$
V_{d}=\frac{1}{d !} \sqrt{\frac{d+1}{2^{d}}}
$$

and all dihedral angles are given by the constant value

$$
\cos \theta_{d}=\frac{1}{d}
$$

so that for example in four dimensions one has $\theta_{d}=\arccos (1 / 4) \approx 75.5^{\circ}$. Local curvatures are then determined by how many simplices $n_{s}(h)$ meet on a given hinge,

$$
\delta(h)=2 \pi-n_{s}(h) \theta_{d}
$$

The action contribution from a single hinge is therefore from $(86) \delta(h) A(h)=$ $\frac{1}{4} \sqrt{3}\left[2 \pi-n_{s}(h) \theta_{d}\right]$ with $n_{s}$ a positive integer. In this model the local curvatures are inherently discrete, and there is no equivalent lattice notion of continuous diffeomorphisms, or for that matter of continuous local deformations corresponding, for example, to shear waves. Indeed it seems rather problematic in this approach to make contact with the continuum theory, as the model does not contain a metric, at least not in an explicit way. This fact has some consequences for the functional measure, since there is really no clear criterion which could be used to restrict it to the form suggested by invariance arguments, as detailed earlier in the discussion of the continuum functional integral for gravity. The hope is that for lattices made of some large number of simplices one would recover some sort of discrete version of diffeomorphism invariance. Recent attempts have focused on simulating the Lorentzian case, but new difficulties arise in this case as it leads in principle to complex weights in the functional integral, which are next to impossible to handle correctly in numerical simulations (since the latter generally rely on positive probabilities).

Another lattice approach somewhat related to the Regge theory described in this review is based on the so-called spin foam models, which have their origin in an observation found in Ponzano and Regge relating the geometry of simplicial lattices to the 
Fig. 5 A four-dimensional hypercube divided up into four-simplices

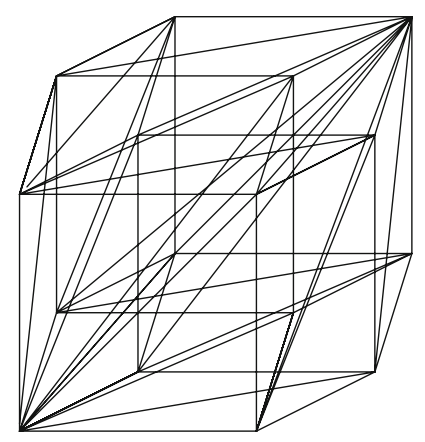

asymptotics of Racah angular momentum addition coefficients. The original concepts were later developed into a spin model for gravity based on quantum spin variables attached to lattice links. In these models representations of SU(2) label edges. One natural underlying framework for such theories is the canonical $3+1$ approach to quantum gravity, wherein quantum spin variables are naturally related to $S U(2)$ spin connections. Extensions to four dimensions have been attempted, and we refer the reader to recent reviews of spin foam models.

\section{Lattice weak field expansion and transverse-traceless modes}

One of the simplest possible problems that can be treated in quantum Regge gravity is the analysis of small fluctuations about a fixed flat Euclidean simplicial background. In this case one finds that the lattice graviton propagator in a De Donder-like gauge is precisely analogous to the continuum expression. To compute an expansion of the lattice Regge action

$$
I_{R} \propto \sum_{\text {hinges }} \delta(l) A(l)
$$

to quadratic order in the lattice weak fields one needs second variations with respect to the edge lengths. The second variation about flat space is given by

$$
\delta^{2} I_{R} \propto \sum_{\text {hinges }}\left(\sum_{\text {edges }} \frac{\partial \delta}{\partial l} \delta l\right) \cdot\left(\sum_{\text {edges }} \frac{\partial A}{\partial l} \delta l\right)
$$

Next a specific lattice structure needs to be chosen as a background geometry. A natural choice is to use a flat hypercubic lattice, made rigid by introducing face diagonals, body diagonals and hyperbody diagonals, which results into a subdivision of each hypercube into $d$ ! (here 4 ! =24) simplices. This particular subdivision is shown in Fig. 5.

By a simple translation, the whole lattice can then be constructed from this one elemental hypercube. Consequently there will be $2^{d}-1=15$ lattice fields per point, 
corresponding to all the edge lengths emanating in the positive lattice directions from any one vertex. Note that the number of degrees per lattice point is slightly larger than what one would have in the continuum, where the metric $g_{\mu \nu}(x)$ has $d(d+1) / 2=10$ degrees of freedom per spacetime point $x$ in four dimensions (perturbatively, the physical degrees of freedom in the continuum are much less: $\frac{1}{2} d(d+1)-1-d-$ $(d-1)=\frac{1}{2} d(d-3)$, for a traceless symmetric tensor, and after imposing gauge conditions). Thus in four dimensions each lattice hypercube will contain four body principals, six face diagonals, four body diagonals and one hyperbody diagonal. Within a given hypercube it is quite convenient to label the coordinates of the vertices using a binary notation, so that the four body principals with coordinates $(1,0,0,0)(0,0,0,1)$ will be labeled by integers $1,2,4,8$, and similarly for the other vertices (thus for example the vertex $(0,1,1,0)$, corresponding to a face diagonal along the second and third Cartesian direction, will be labeled by the integer 6 ).

For a given lattice of fixed connectivity, the edge lengths are then allowed to fluctuate around an equilibrium value $l_{i}^{0}$

$$
l_{i}=l_{i}^{0}\left(1+\epsilon_{i}\right)
$$

In the case of the hypercubic lattice subdivided into simplices, the unperturbed edge lengths $l_{i}^{0}$ take on the values $1, \sqrt{2}, \sqrt{3}, 2$, depending on edge type. The second variation of the action then reduces to a quadratic form in the 15-component small fluctuation vector $\epsilon_{n}$

$$
\delta^{2} I_{R} \propto \sum_{m n} \epsilon_{m}^{T} M_{m n} \epsilon_{n}
$$

Here $M$ is the small fluctuation matrix, whose inverse determines the free lattice graviton propagator, and the indices $m$ and $n$ label the sites on the lattice. But just as in the continuum, $M$ has zero eigenvalues and cannot therefore be inverted until one supplies an appropriate gauge condition. Specifically, one finds that the matrix $M$ in four dimensions has four zero modes corresponding to periodic translations of the lattice, and a fifth zero mode corresponding to periodic fluctuations in the hyperbody diagonal. After block-diagonalization it is found that four modes completely decouple and are constrained to vanish, and thus the remaining degrees of freedom are 10, as in the continuum, where the metric has ten independent components. The wrong sign for the conformal mode, which is present in the continuum, is also reproduced by the lattice propagator.

Due to the locality of the original lattice action, the matrix $M$ can be considered local as well, since it only couples edge fluctuations on neighboring lattice sites. In Fourier space one can write for each of the fifteen displacements $\epsilon_{n}^{i+j+k+l}$, defined at the vertex of the hypercube with labels $(i, j, k, l)$,

$$
\epsilon_{n}^{i+j+k+l}=\left(\omega_{1}\right)^{i}\left(\omega_{2}\right)^{j}\left(\omega_{4}\right)^{k}\left(\omega_{8}\right)^{l} \epsilon_{n}^{0}
$$

with $\omega_{1}=e^{i k_{1}}, \omega_{2}=e^{i k_{2}}, \omega_{4}=e^{i k_{3}}$ and $\omega_{8}=e^{i k_{4}}$ (it will be convenient in the following to use binary notation for $\omega$ and $\epsilon$, but the regular notation for $k_{i}$ ). Here 
and in the following we have set the lattice spacing $a$ equal to one. The remaining dynamics is encoded in the $10 \times 10$ dimensional matrix $L_{\omega}=A_{10}-\frac{1}{18} B B^{\dagger}$. By a second rotation, here affected by a matrix $T$, it can finally be brought into the form

$$
\tilde{L}_{\omega}=T^{\dagger} L_{\omega} T=[8-(\Sigma+\bar{\Sigma})]\left(\begin{array}{cc}
\frac{1}{2} \beta & 0 \\
0 & I_{6}
\end{array}\right)-C^{\dagger} C
$$

with the matrix $\beta$ given by

$$
\beta=\frac{1}{2}\left(\begin{array}{cccc}
1 & -1 & -1 & -1 \\
-1 & 1 & -1 & -1 \\
-1 & -1 & 1 & -1 \\
-1 & -1 & -1 & 1
\end{array}\right)
$$

The other matrix $C$ appearing in the second term is given by

$$
C=\left(\begin{array}{cccccccccc}
f_{1} & 0 & 0 & 0 & \tilde{f}_{2} & \tilde{f}_{4} & 0 & \tilde{f}_{8} & 0 & 0 \\
0 & f_{2} & 0 & 0 & \tilde{f}_{1} & 0 & \tilde{f}_{4} & 0 & \tilde{f}_{8} & 0 \\
0 & 0 & f_{4} & 0 & 0 & \tilde{f}_{1} & \tilde{f}_{2} & 0 & 0 & \tilde{f}_{8} \\
0 & 0 & 0 & f_{8} & 0 & 0 & 0 & \tilde{f}_{1} & \tilde{f}_{2} & \tilde{f}_{4}
\end{array}\right)
$$

with $f_{i} \equiv \omega_{i}-1$ and $\tilde{f}_{i} \equiv 1-\bar{\omega}_{i}$. Furthermore $\Sigma=\sum_{i} \omega_{i}$, and for small momenta one finds

$$
8-(\Sigma+\bar{\Sigma})=8-\sum_{i=1}^{4}\left(e^{i k_{i}}+e^{-i k_{i}}\right) \sim k^{2}+O\left(k^{4}\right)
$$

which shows that the surviving terms in the lattice action are indeed quadratic in $k$. At this point one is finally ready for a comparison with the continuum result, namely with the Lagrangian for pure gravity in the weak field limit, namely

$$
\begin{aligned}
\mathcal{L}_{\mathrm{sym}}= & -\frac{1}{2} \partial_{\lambda} h_{\lambda \mu} \partial_{\mu} h_{\nu v}+\frac{1}{2} \partial_{\lambda} h_{\lambda \mu} \partial_{\nu} h_{\nu \mu} \\
& -\frac{1}{4} \partial_{\lambda} h_{\mu \nu} \partial_{\lambda} h_{\mu \nu}+\frac{1}{4} \partial_{\lambda} h_{\mu \mu} \partial_{\lambda} h_{\nu \nu}
\end{aligned}
$$

The latter can be conveniently split into two parts, as follows

$$
\mathcal{L}_{\text {sym }}=-\frac{1}{2} \partial_{\lambda} h_{\alpha \beta} V_{\alpha \beta \mu \nu} \partial_{\lambda} h_{\mu \nu}+\frac{1}{2} C^{2}
$$

with

$$
V_{\alpha \beta \mu \nu}=\frac{1}{2} \eta_{\alpha \mu} \eta_{\beta \nu}-\frac{1}{4} \eta_{\alpha \beta} \eta_{\mu \nu}
$$


with metric components $11,22,33,44,12,13,14,23,24,34$ more conveniently labeled sequentially by integers $1, \ldots, 10$. The gauge fixing term $C_{\mu}$ is

$$
C_{\mu}=\partial_{\nu} h_{\mu \nu}-\frac{1}{2} \partial_{\mu} h_{\nu v}
$$

The above expression is still not quite the same as the lattice weak field action, but a simple transformation to trace reversed variables $\bar{h}_{\mu \nu} \equiv h_{\mu \nu}-\frac{1}{2} \delta_{\mu \nu} h_{\lambda \lambda}$ leads to

$$
\mathcal{L}_{\mathrm{sym}}=\frac{1}{2} k_{\lambda} \bar{h}_{i} V_{i j} k_{\lambda} \bar{h}_{j}-\frac{1}{2} \bar{h}_{i}\left(C^{\dagger} C\right)_{i j} \bar{h}_{j}
$$

with the matrix $V$ given by

$$
V_{i j}=\left(\begin{array}{cc}
\frac{1}{2} & 0 \\
0 & I_{6}
\end{array}\right)
$$

with $k=i \partial$. Now $\beta$ is the same as the matrix in (141), and $C$ is nothing but the small $k$ limit of the matrix by the same name in (142).

It is easy to see that the sequence of transformations expressed by the matrices $S$ and $T$ relating the lattice fluctuations $\epsilon_{i}(n)$ to their continuum counterparts $h_{\mu \nu}(x)$, just reproduces the expected relationship between lattice and continuum fields. On the one hand one has $g_{\mu \nu}=\eta_{\mu \nu}+h_{\mu \nu}$, where $\eta_{\mu \nu}$ is the flat metric. At the same time one has from (54) for each simplex within a given hypercube

$$
g_{i j}=\frac{1}{2}\left(l_{0 i}^{2}+l_{0 j}^{2}-l_{i j}^{2}\right)
$$

By inserting $l_{i}=l_{i}^{0}\left(1+\epsilon_{i}\right)$, with $l_{i}^{0}=1, \sqrt{2}, \sqrt{3}, 2$ for the body principal $(i=1,2$, $4,8)$, face diagonal $(i=3,5,6,9,10,12)$, body diagonal $(i=7,11,13,14)$ and hyperbody diagonal $(i=15)$, respectively, one gets for example $\left(1+\epsilon_{1}\right)^{2}=1+h_{11}$, $\left(1+\epsilon_{3}\right)^{2}=1+\frac{1}{2}\left(h_{11}+h_{22}\right)+h_{12}$, etc., which in turn can then be solved for the $\epsilon$ 's in terms of the $h_{\mu \nu}$ 's,

$$
\begin{aligned}
\epsilon_{1}= & \frac{1}{2} h_{11}+O\left(h^{2}\right) \\
\epsilon_{3}= & \frac{1}{2} h_{12}+\frac{1}{4}\left(h_{11}+h_{22}\right)+O\left(h^{2}\right) \\
\epsilon_{7}= & \frac{1}{6}\left(h_{12}+h_{13}+h_{23}\right)+\frac{1}{6}\left(h_{23}+h_{13}+h_{12}\right) \\
& +\frac{1}{6}\left(h_{11}+h_{22}+h_{33}\right)+O\left(h^{2}\right)
\end{aligned}
$$

and so on. As expected, the lattice action has a local gauge invariance, whose explicit form in the weak field limit can be obtained explicitly. This continuous local invariance has $d$ parameters in $d$ dimensions and describes therefore lattice diffeomorphisms. In 
the quantum theory, such local gauge invariance implies the existence of Ward identities for $n$-point functions.

\section{Strong coupling expansion}

In this section the strong coupling (large $G$ or small $k=1 /(8 \pi G)$ ) expansion of the lattice gravitational functional integral will be discussed. The resulting series is in general expected to be useful up to some $k=k_{c}$, where $k_{c}$ is the lattice critical point, at which the partition function develops a singularity. One starts from the lattice regularized path integral with action (89) and measure (94). Then the four-dimensional Euclidean lattice action contains the usual cosmological constant and Regge scalar curvature terms

$$
I_{\text {latt }}=\lambda \sum_{h} V_{h}\left(l^{2}\right)-k \sum_{h} \delta_{h}\left(l^{2}\right) A_{h}\left(l^{2}\right),
$$

with $k=1 /(8 \pi G)$, and possibly additional higher derivative terms as well. The action only couples edges which belong either to the same simplex or to a set of neighboring simplices, and can therefore be considered as local, just like the continuum action. It leads to a lattice partition function defined in (96)

$$
Z_{\text {latt }}=\int\left[d l^{2}\right] e^{-\lambda_{0} \sum_{h} V_{h}+k \sum_{h} \delta_{h} A_{h}},
$$

where, as customary, the lattice ultraviolet cutoff is set equal to one (i.e., all length scales are measured in units of the lattice cutoff). For definiteness the measure will be of the form

$$
\int\left[d l^{2}\right]=\int_{0}^{\infty} \prod_{s}\left(V_{d}(s)\right)^{\sigma} \prod_{i j} d l_{i j}^{2} \Theta\left[l_{i j}^{2}\right]
$$

When doing an expansion in the kinetic term proportional to $k$, it is convenient to include the $\lambda$-term in the measure. We will set therefore here as in (98)

$$
d \mu\left(l^{2}\right) \equiv\left[d l^{2}\right] e^{-\lambda_{0} \sum_{h} V_{h}}
$$

As a next step, $Z_{\text {latt }}$ is expanded in powers of $k$,

$$
Z_{\text {latt }}(k)=\int d \mu\left(l^{2}\right) e^{k \sum_{h} \delta_{h} A_{h}}=\sum_{n=0}^{\infty} \frac{1}{n !} k^{n} \int d \mu\left(l^{2}\right)\left(\sum_{h} \delta_{h} A_{h}\right)^{n} .
$$

It is easy to show that $Z(k)=\sum_{n=0}^{\infty} a_{n} k^{n}$ is analytic at $k=0$, so this expansion should be well defined up to the nearest singularity in the complex $k$ plane. One key 
quantity in the strong coupling expansion of lattice gravity is the correlation between different plaquettes,

$$
\left\langle(\delta A)_{h}(\delta A)_{h^{\prime}}\right\rangle=\frac{\int d \mu\left(l^{2}\right)(\delta A)_{h}(\delta A)_{h^{\prime}} e^{k \sum_{h} \delta_{h} A_{h}}}{\int d \mu\left(l^{2}\right) e^{k \sum_{h} \delta_{h} A_{h}}},
$$

or, better, its connected part (denoted here by $\langle\cdots\rangle_{C}$ ). Here again the exponentials in the numerator and denominator can be expanded out in powers of $k$. The lowest order term in $k$ will involve the correlation

$$
\int d \mu\left(l^{2}\right)(\delta A)_{h}(\delta A)_{h^{\prime}}
$$

But unless the two hinges are close to each other, they will fluctuate in an uncorrelated manner, with $\left\langle(\delta A)_{h}(\delta A)_{h^{\prime}}\right\rangle-\left\langle(\delta A)_{h}\right\rangle\left\langle(\delta A)_{h^{\prime}}\right\rangle=0$. In order to achieve a non-trivial correlation, the path between the two hinges $h$ and $h^{\prime}$ needs to be tiled by at least as many terms from the product $\left(\sum_{h} \delta_{h} A_{h}\right)^{n}$ in

$$
\int d \mu\left(l^{2}\right)(\delta A)_{h}(\delta A)_{h^{\prime}}\left(\sum_{h} \delta_{h} A_{h}\right)^{n}
$$

as are needed to cover the distance $l$ between the two hinges. One then has

$$
\left\langle(\delta A)_{h}(\delta A)_{h^{\prime}}\right\rangle_{C} \sim k^{l} \sim e^{-l / \xi},
$$

with the correlation length $\xi=1 /|\log k| \rightarrow 0$ to lowest order as $k \rightarrow 0$ (here we have used the usual definition of the correlation length $\xi$, namely that a generic correlation function is expected to decay as $\exp (-$ distance $/ \xi$ ) for large separations). This last result is quite general, and holds for example irrespective of the boundary conditions (unless of course $\xi \sim L$, where $L$ is the linear size of the system, in which case a path can be found which wraps around the lattice). But further thought reveals that the above result is in fact not completely correct, due to the fact that in order to achieve a non-vanishing correlation one needs, at least to lowest order, to connect the two hinges by a narrow tube. The previous result should then read correctly as

$$
\left\langle(\delta A)_{h}(\delta A)_{h^{\prime}}\right\rangle_{C} \sim\left(k^{n_{d}}\right)^{l},
$$

where $n_{d} l$ represents the minimal number of dual lattice polygons needed to form a closed surface connecting the hinges $h$ and $h^{\prime}$, with $l$ the actual distance (in lattice units) between the two hinges. Figure 6 provides an illustration of the situation.

With some additional effort many additional terms can be computed in the strong coupling expansion. In practice the method is generally not really competitive with direct numerical evaluation of the path integral via Monte Carlo methods. But it does provide a new way of looking at the functional integral, and provide the basis for new approaches, such as the large $d$ limit to be discussed in the second half of the next section. 


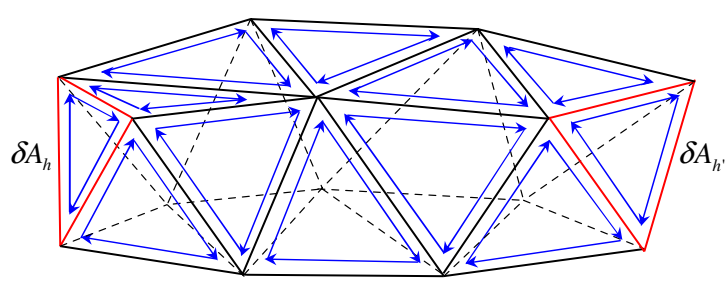

Fig. 6 Correlations between action contributions on hinge $h$ and hinge $h^{\prime}$ arise to lowest order in the strong coupling expansions from diagrams describing a narrow tube connecting the two hinges. Here vertices represent points in the dual lattice, with the tube-like closed surface tiled with parallel transport polygons. For each link of the dual lattice, the $\mathrm{SO}(4)$ parallel transport matrices $\mathbf{R}$ are represented by an arrow

\section{Gravitational Wilson loop}

An important question for any theory of quantum gravity is what gravitational observables should look like, i.e., which expectation values of operators (or ratios thereof) have meaning and physical interpretation in the context of a manifestly covariant formulation, in particular in a situation where metric fluctuations are not necessarily bounded. Such averages naturally include the previously discussed expectation values of the (integrated) scalar curvature and other related quantities (involving for example curvature-squared terms), as well as correlations of operators at fixed geodesic distance. Another set of physical observables on which we focus here corresponds to the gravitational analog of the Wilson loop. It provides information about the parallel transport of vectors, and therefore on the effective curvature, around large, near-planar loops. In contrast to gauge theories, the Wilson loop in quantum gravity does not give information on the static potential, which is obtained instead for the correlation between particle world-lines.

The gauge theory definition can be adapted to the lattice gravitational case. It turns out that it is most easily achieved by using a slight variant of Regge calculus, in which the action coincides with the usual Regge action in the near-flat limit. Here we will use extensively the notion of lattice parallel transport discussed earlier, and how areas are defined on the dual lattice.

At strong coupling the measure and cosmological constant terms form the dominant part of the functional integral, since the Einstein part of the action is vanishingly small in this limit. Yet, and in contrast to strongly coupled lattice Yang-Mills theories, the functional integral is still non-trivial to compute analytically in this limit, mainly due to the triangle inequality constraints. Therefore, in order to be able to derive some analytical estimates for correlation functions in the strong coupling limit, one needs still to develop some set of approximation methods. In principle the reliability of the approximations can later be tested by numerical means, for example by integrating directly over edges using the explicit lattice measure given above.

One approach that appears natural in the gravity context follows along the lines of what is normally done in gauge theories, namely an integration over compact group variables, using the invariant measure over the gauge group. It is of this method that we wish to take advantage here, as we believe that it is well suited for gravity as well. In order to apply such a technique to gravity one needs (i) to formulate the lattice 
theory in such a way that group variables are separated and therefore appear explicitly; (ii) integrate over the group variables using an invariant measure; and (iii) approximate the relevant correlation functions in such a way that the group integration can be performed exactly, using for example mean field methods for the parts that appear less tractable. In such a program one is aided by the fact that in the strong coupling limit one is expanding about a well defined ground state, and that the measure and the interactions are local, coupling only lattice variable (edges or rotations) which are a few lattice spacings apart. The down side of such methods is that one is no longer evaluating the functional integral for quantum gravity exactly, even in the strong coupling limit; the upside is that one obtains a clear analytical estimate, which later can be in principle systematically tested by numerical methods (which are in principle exact).

In the gravity case the analogs of the gauge variables of Yang-Mills theories are given by the connection, so it is natural therefore to look for a first order formulation of Regge gravity. The main feature of this approach is that one treats the metric $g_{\mu \nu}$ and the affine connection $\Gamma_{\mu \nu}^{\lambda}$ as independent variables. There one can safely consider functionally integrating separately over the affine connection and the metric, treated as independent variables, with the correct relationship between metric and connection arising then as a consequence of the dynamics. In the lattice theory we will follow a similar spirit, separating out explicitly in the lattice action the degrees of freedom corresponding to local rotations (the analogs of the $\Gamma$ 's in the continuum), which we will find to be most conveniently described by orthogonal matrices $\mathbf{R}$.

The next step is a use of the properties of local rotation matrices in the context of the lattice theory, and how these relate to the lattice gravitational action. It was shown earlier that with each neighboring pair of simplices $s, s+1$ one can associate a Lorentz transformation $R^{\mu}{ }_{v}(s, s+1)$. For a closed elementary path $C_{h}$ encircling a hinge $h$ and passing through each of the simplices that meet at that hinge one has for the total rotation matrix $\mathbf{R} \equiv \prod_{s} R_{s, s+1}$ associated with the given hinge

$$
\left[\prod_{s} R_{s, s+1}\right]_{v}^{\mu}=\left[e^{\delta(h) U(h)}\right]_{\nu}^{\mu},
$$

as in (80). More generally one might want to consider a near-planar, but non-infinitesimal, closed loop $C$, as shown in Fig. 7. Along this closed loop the overall rotation matrix will still be given by

$$
R_{\nu}^{\mu}(C)=\left[\prod_{s \subset C} R_{s, s+1}\right]_{\nu}^{\mu}
$$

In analogy with the infinitesimal loop case, one would like to state that for the overall rotation matrix one has

$$
R_{\nu}^{\mu}(C) \approx\left[e^{\delta(C) U(C))}\right]_{\nu}^{\mu}
$$

where $U_{\mu \nu}(C)$ is now an area bivector perpendicular to the loop, which will work only if the loop is close to planar so that $U_{\mu \nu}$ can be taken to be approximately constant 


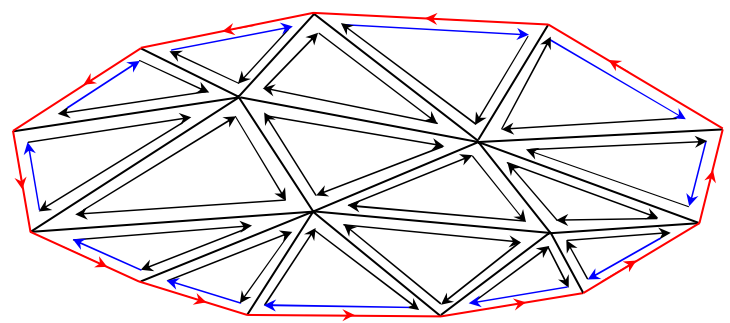

Fig. 7 Gravitational analog of the Wilson loop. A vector is parallel-transported along the larger outer loop. The enclosed minimal surface is tiled with parallel transport polygons, here chosen to be triangles for illustrative purposes. For each link of the dual lattice, the elementary parallel transport matrices $\mathbf{R}\left(s, s^{\prime}\right)$ are represented by arrows. In spite of the fact that the (Lorentz) matrices $\mathbf{R}$ can fluctuate strongly in accordance with the local geometry, two contiguous, oppositely oriented arrows always give $\mathbf{R R}^{-1}=1$

along the path $C$. By a near-planar loop around the point $P$, we mean one that is constructed by drawing outgoing geodesics, on a plane through $P$.

If that is true, then one can define an appropriate coordinate scalar by contracting the above rotation matrix $\mathbf{R}(C)$ with the some appropriate bivector, namely

$$
W(C)=\omega_{\alpha \beta}(C) R^{\alpha \beta}(C)
$$

where the bivector, $\omega_{\alpha \beta}(C)$, is intended as being representative of the overall geometric features of the loop (for example, it can be taken as an average of the hinge bivector $\omega_{\alpha \beta}(h)$ along the loop).

In the quantum theory one is interested in the average of the above loop operator $W(C)$. Now one notes that for any continuum manifold one can define locally the parallel transport of a vector around a near-planar loop $C$. If the curvature of the manifold is small, one can treat the larger loop the same way as the small one; then the expression of (164) for the rotation matrix $\mathbf{R}(C)$ associated with a near-planar loop can be re-written in terms of a surface integral of the large-scale Riemann tensor, projected along the surface area element bivector $A^{\alpha \beta}(C)$ associated with the loop,

$$
R_{\nu}^{\mu}(C) \approx\left[e^{\frac{1}{2} \int_{S} R^{\cdot} \cdot \alpha \beta} A^{\alpha \beta}(C)\right]_{\nu}^{\mu} .
$$

Thus a direct calculation of the Wilson loop provides a way of determining the effective curvature at large distance scales, even in the case where short distance fluctuations in the metric may be significant.

A detailed lattice calculation at strong coupling then gives the following result. First one defines the lattice Wilson loop as

$$
W(C)=\left\langle\operatorname{Tr}\left[\left(U_{C}+\epsilon I_{4}\right) R_{1} R_{2} \ldots R_{n}\right]\right\rangle .
$$

where the $R_{i}$ 's are the rotation matrices along the path and the factor $\left(U_{C}+\epsilon I_{4}\right)$, containing some "average" direction bivector, $U_{C}$, for the loop, which, after all, is assumed to be almost planar. For sufficiently strong coupling one obtains an area law, 
in other words the above quantity behaves for large areas as

$$
\exp \left[\left(A_{C} / \bar{A}\right) \log (k \bar{A} / 16)\right]=\exp \left(-A_{C} / \xi^{2}\right)
$$

where $\xi \equiv[\bar{A} /|\log (k \bar{A} / 16)|]^{1 / 2}$. The rapid decay of the quantum gravitational Wilson loop as a function of the area is seen as a general and direct consequence of the assumed disorder in the uncorrelated fluctuations of the parallel transport matrices $\mathbf{R}\left(s, s^{\prime}\right)$ at strong coupling.

Here it is important to note that the gravitational correlation length $\xi$ is defined independently of the expectation value of the Wilson loop. Indeed a key quantity in gauge theories as well as gravity is the correlation between different plaquettes, which in simplicial gravity is given by [see (157)],

$$
\left\langle(\delta A)_{h}(\delta A)_{h^{\prime}}\right\rangle=\frac{\int d \mu\left(l^{2}\right)(\delta A)_{h}(\delta A)_{h^{\prime}} e^{k \sum_{h} \delta_{h} A_{h}}}{\int d \mu\left(l^{2}\right) e^{k \sum_{h} \delta_{h} A_{h}}} .
$$

The final step is an interpretation of this last result in semi-classical terms. As discussed at the beginning of this section, the rotation matrix appearing in the gravitational Wilson loop can be related classically to a well-defined physical process: a vector is parallel transported around a large loop, and at the end it is compared to its original orientation. The vector's rotation is then directly related to some sort of average curvature enclosed by the loop. The total rotation matrix $\mathbf{R}(C)$ is given in general by a path-ordered $(\mathcal{P})$ exponential of the integral of the affine connection $\Gamma_{\mu \nu}^{\lambda}$ via

$$
R_{\beta}^{\alpha}(C)=\left[\mathcal{P} \exp \left\{\oint_{\text {path C }} \Gamma_{\lambda} \cdot d x^{\lambda}\right\}\right]_{\beta}^{\alpha} .
$$

In such a semi classical description of the parallel transport process of a vector around a very large loop, one can re-express the connection in terms of a suitable coarsegrained, or semi-classical, Riemann tensor, using Stokes' theorem

$$
R_{\beta}^{\alpha}(C) \sim\left[\exp \left\{\frac{1}{2} \int_{S(C)} R^{\cdot} \cdot \mu \nu A_{C}^{\mu \nu}\right\}\right]_{\beta}^{\alpha},
$$

where here $A_{C}^{\mu \nu}$ is the usual area bivector associated with the loop in question. The use of semi-classical arguments in relating the above rotation matrix $\mathbf{R}(C)$ to the surface integral of the Riemann tensor assumes (as usual in the classical context) that the curvature is slowly varying on the scale of the very large loop. Since the rotation is 
small for weak curvatures, one can write

$$
R_{\beta}^{\alpha}(C) \sim\left[1+\frac{1}{2} \int_{S(C)} R^{\cdot} \cdot \mu \nu A_{C}^{\mu \nu}+\cdots\right]_{\beta}^{\alpha} .
$$

At this stage one is ready to compare the above expression to the quantum result of (168). Since one expression (172) is a matrix and the other (168) is a scalar, we shall take the trace after first contracting the rotation matrix with $\left(U_{C}+\epsilon I_{4}\right)$, as in the definition of the Wilson loop, giving

$$
W(C) \sim \operatorname{Tr}\left(\left(U_{C}+\epsilon I_{4}\right) \exp \left\{\frac{1}{2} \int_{S(C)} R^{\cdot} \cdot{ }_{\mu \nu} A_{C}^{\mu \nu}\right\}\right) .
$$

For the lattice analog of a background manifold with constant or near-constant large scale curvature one has

$$
\begin{aligned}
R_{\mu \nu \lambda \sigma} & =\frac{1}{3} \lambda\left(g_{\mu \nu} g_{\lambda \sigma}-g_{\mu \lambda} g_{\nu \sigma}\right) \\
R_{\mu \nu \lambda \sigma} R^{\mu \nu \lambda \sigma} & =\frac{8}{3} \lambda^{2}
\end{aligned}
$$

so that here one can set

$$
R_{\beta \mu \nu}^{\alpha}=\bar{R} U_{\beta}^{\alpha} U_{\mu \nu},
$$

where $\bar{R}$ is some average curvature over the loop, and the $U$ 's here will be taken to coincide with $U_{C}$. The trace of the product of $\left(U_{C}+\epsilon I_{4}\right)$ with this expression gives

$$
\operatorname{Tr}\left(\bar{R} U_{C}^{2} A_{C}\right)=-2 \bar{R} A_{C},
$$

where one has used $U_{\mu \nu} A_{C}^{\mu \nu}=2 A_{C}$ (the choice of direction for the bivectors will be such that the latter is true for all loops). This is to be compared with the linear term from the other exponential expression, $-A_{C} / \xi^{2}$. Thus the average curvature is computed to be of the order

$$
\bar{R} \sim 1 / \xi^{2}
$$

at least in the small $k=1 / 8 \pi G$ limit. An equivalent way of phrasing the last result is that $1 / \xi^{2}$ should be identified, up to a constant of proportionality, with the scaled cosmological constant $\lambda$. 


\section{Nonperturbative gravity}

The exact evaluation of the lattice functional integral for quantum gravity by numerical methods allows one to investigate a regime which is generally inaccessible by perturbation theory, where the coupling $G$ is strong and quantum fluctuations in the metric are expected to be large. The hope in the end is to make contact with the analytic results obtained, for example, in the $2+\epsilon$ expansion, and determine which scenarios are physically realized in the lattice regularized model, and then perhaps even in the real world.

Specifically, one can enumerate several major questions that one would like to get at least partially answered. The first one is: which scenarios suggested by perturbation theory are realized in the lattice theory? Perhaps a stable ground state for the quantum theory cannot be found, which would imply that the regulated theory is still inherently pathological. Furthermore, if a stable ground state exists for some range of bare parameters, does it require the inclusion of higher derivative couplings in an essential way, or is the minimal theory, with an Einstein and a cosmological term, sufficient? Does the presence of dynamical matter, say in the form of a massless scalar field, play an important role, or is the non-perturbative dynamics of gravity determined largely by the pure gravity sector (as in Yang-Mills theories)?

More generally, is there any indication that the non-trivial ultraviolet fixed point scenario is realized in the lattice theory in four dimensions? This would imply, as in the non-linear sigma model, the existence of at least two physically distinct phases and non-trivial exponents. Which quantity can be used as an order parameter to physically describe, in a qualitative, way the two phases? A clear physical characterization of the two phases would allow one, at least in principle, to decide which phase, if any, could be realized in nature. Ultimately this might or might not be possible based on purely qualitative aspects. As will discussed below, the lattice continuum limit is taken in the vicinity of the fixed point, so close to it is the physically most relevant regime. At the next level one would hope to be able to establish a quantitative connection with those continuum perturbative results which are not affected by uncontrollable errors, such as for example the $2+\epsilon$ expansion discussed earlier. Since the lattice cutoff and the method of dimensional regularization cut the theory off in the ultraviolet in rather different ways, one needs to compare universal quantities which are cutoff-independent. One example is the critical exponent $v$, as well as any other non-trivial scaling dimension that might arise. Within the $2+\epsilon$ expansion only one such exponent appears, to all orders in the loop expansion, as $v^{-1}=-\beta^{\prime}\left(G_{c}\right)$. Therefore one central issue in the lattice regularized theory is the value of the universal exponent $v$.

Knowledge of $v$ would allow one to be more specific about the running of the gravitational coupling. One purpose of the earlier discussion was to convince the reader that the exponent $v$ determines the renormalization group running of $G\left(\mu^{2}\right)$ in the vicinity of the fixed point, as in (46) for quantized gravity. From a practical point of view, on the lattice it is difficult to determine the running of $G\left(\mu^{2}\right)$ directly from correlation functions, since the effects from the running of $G$ are generally small. Instead one would like to make use of the analog of (50) for gravity to determine $v$, and from there the running of $G$. But the correlation length $\xi=m^{-1}$ is also difficult to compute, since it enters the curvature correlations at fixed geodesic distance, which are 
hard to compute for (genuinely geometric) reasons to be discussed later. Furthermore, these generally decay exponentially in the distance at strong $G$, and can therefore be difficult to compute due to the signal to noise problem of numerical simulations. Fortunately the exponent $v$ can be determined instead, and with good accuracy, from singularities of the derivatives of the path integral $Z$, whose singular part is expected, on the basis of very general arguments, to behave in the vicinity of the fixed point as $F \equiv-\frac{1}{V} \ln Z \sim \xi^{-d}$ where $\xi$ is the gravitational correlation length. From (50) relating $\xi(G)$ to $G-G_{c}$ and $\nu$ one can then determine $\nu$, as well as the critical coupling $G_{c}$.

The starting point is once again the lattice regularized path integral with action as in (89) and measure as in (94),

$$
Z_{\text {latt }}=\int\left[d l^{2}\right] e^{-\lambda_{0} \sum_{h} V_{h}+k \sum_{h} \delta_{h} A_{h}},
$$

where, as customary, the lattice ultraviolet cutoff is set equal to one (i.e., all length scales are measured in units of the lattice cutoff). The lattice measure is given in (94) and is therefore of the form

$$
\int\left[d l^{2}\right]=\int_{0}^{\infty} \prod_{s}\left(V_{d}(s)\right)^{\sigma} \prod_{i j} d l_{i j}^{2} \Theta\left[l_{i j}^{2}\right] .
$$

with $\sigma$ a real parameter. Ultimately the above lattice partition function $Z_{\text {latt }}$ is intended as a regularized form of the continuum Euclidean Feynman path integral of (14).

Among the simplest quantum mechanical averages is the one associated with the local curvature

$$
\mathcal{R}(k) \sim \frac{\left\langle\int d x \sqrt{g} R(x)\right\rangle}{\left\langle\int d x \sqrt{g}\right\rangle},
$$

The curvature associated with the quantity above is the one that would be detected when parallel-transporting vectors around infinitesimal loops, with size comparable to the average lattice spacing $l_{0}$. Closely related to it is the fluctuation in the local curvature

$$
\chi_{\mathcal{R}}(k) \sim \frac{\left\langle\left(\int d x \sqrt{g} R\right)^{2}\right\rangle-\left\langle\int d x \sqrt{g} R\right\rangle^{2}}{\left\langle\int d x \sqrt{g}\right\rangle} .
$$

The latter is related to the connected curvature correlation at zero momentum

$$
\chi_{\mathcal{R}} \sim \frac{\int d x \int d y\langle\sqrt{g(x)} R(x) \sqrt{g(y)} R(y)\rangle_{c}}{\left\langle\int d x \sqrt{g(x)}\right\rangle} .
$$

Both $\mathcal{R}(k)$ and $\chi_{\mathcal{R}}(k)$ are directly related to derivatives of $Z$ with respect to $k$,

$$
\mathcal{R}(k) \sim \frac{1}{V} \frac{\partial}{\partial k} \ln Z
$$


and

$$
\chi_{\mathcal{R}}(k) \sim \frac{1}{V} \frac{\partial^{2}}{\partial k^{2}} \ln Z .
$$

Thus a divergence or non-analyticity in $Z$, as caused for example by a phase transition, is expected to show up in these local averages as well. Note that the above expectation values are manifestly invariant, since they are related to derivatives of $Z$.

When computing correlations, new issues arise in quantum gravity due to the fact that the physical distance between any two points $x$ and $y$

$$
d(x, y \mid g)=\min _{\xi} \int_{\tau(x)}^{\tau(y)} d \tau \sqrt{g_{\mu \nu}(\xi) \frac{d \xi^{\mu}}{d \tau} \frac{d \xi^{\nu}}{d \tau}},
$$

is a fluctuating function of the background metric $g_{\mu \nu}(x)$. In addition, the Lorentz group used to classify spin states is meaningful only as a local concept. In the continuum the shortest distance between two events is determined by solving the geodesic equation

$$
\frac{d^{2} x^{\mu}}{d \tau^{2}}+\Gamma_{\lambda \sigma}^{\mu} \frac{d x^{\lambda}}{d \tau} \frac{d x^{\sigma}}{d \tau}=0
$$

On the lattice the geodesic distance between two lattice vertices $x$ and $y$ requires the determination of the shortest lattice path connecting several lattice vertices, and having the two given vertices as endpoints. This can be done at least in principle by enumerating all paths connecting the two points, and then selecting the shortest one. Consequently physical correlations have to be defined at fixed geodesic distance $d$, as in the following correlation between scalar curvatures

$$
\left\langle\int d x \int d y \sqrt{g} R(x) \sqrt{g} R(y) \delta(|x-y|-d)\right\rangle
$$

Generally these do not go to zero at large separation, so one needs to define the connected part, by subtracting out the value at $d=\infty$. These will be indicated in the following by the connected \langle\rangle$_{c}$ average, and we will write the resulting connected curvature correlation function at fixed geodesic distance compactly as

$$
G_{R}(d) \sim\langle\sqrt{g} R(x) \sqrt{g} R(y) \delta(|x-y|-d)\rangle_{c} .
$$

One can define several more invariant correlation functions at fixed geodesic distance for other operators involving curvatures. Thus one is naturally lead to the connected correlation function

$$
G_{R}(d) \equiv\left\langle\sum_{h \supset x} \delta_{h} A_{h} \sum_{h^{\prime} \supset y} \delta_{h^{\prime}} A_{h^{\prime}} \delta(|x-y|-d)\right\rangle_{c},
$$

which probes correlations in the scalar curvatures. 
In general one expects for the curvature correlation either a power law decay, for distances sufficiently larger than the lattice spacing $l_{0}$,

$$
\langle\sqrt{g} R(x) \sqrt{g} R(y) \delta(|x-y|-d)\rangle_{c} \underset{d \gg l_{0}}{\sim} \frac{1}{d^{2 n}},
$$

with $n$ some exponent characterizing the power law decay, or at very large distances an exponential decay, characterized by a correlation length $\xi$,

$$
\langle\sqrt{g} R(x) \sqrt{g} R(y) \delta(|x-y|-d)\rangle_{c} \underset{d \gg \xi}{\sim} e^{-d / \xi}
$$

In practice the correlation functions at fixed geodesic distance are difficult to compute numerically, and therefore not the best route to study the critical properties. But scaling arguments allow one to determine the scaling behavior of correlation functions from critical exponents characterizing the singular behavior of the free energy and various local averages in the vicinity of the critical point. In general a divergence of the correlation length $\xi$

$$
\xi(k) \equiv \underset{k \rightarrow k_{c}}{\sim} A_{\xi}\left|k_{c}-k\right|^{-v}
$$

signals the presence of a phase transition, and leads to the appearance of a singularity in the free energy $F(k)$. The scaling assumption for the free energy postulates that a divergent correlation length in the vicinity of the critical point at $k_{c}$ leads to non-analyticities of the type

$$
F \equiv-\frac{1}{V} \ln Z=F_{\text {reg }}+F_{\text {sing }} \quad F_{\text {sing }} \sim \xi^{-d}
$$

where the second relationship follows simply from dimensional arguments (the free energy is an extensive quantity). The regular part $F_{\text {reg }}$ is generally not determined from $\xi$ by purely dimensional considerations, but as the name implies is a regular function in the vicinity of the critical point. Combining the definition of $v$ in (192) with the scaling assumption of (193) one obtains

$$
F_{\text {sing }}(k) \underset{k \rightarrow k_{c}}{\sim}\left|k_{c}-k\right|^{d v}
$$

The presence of a phase transition can then be inferred from non-analytic terms in invariant averages, such as the average curvature and its fluctuation. Thus for the average curvature one obtains

$$
\mathcal{R}(k) \underset{k \rightarrow k_{c}}{\sim} A_{\mathcal{R}}\left|k_{c}-k\right|^{d v-1},
$$




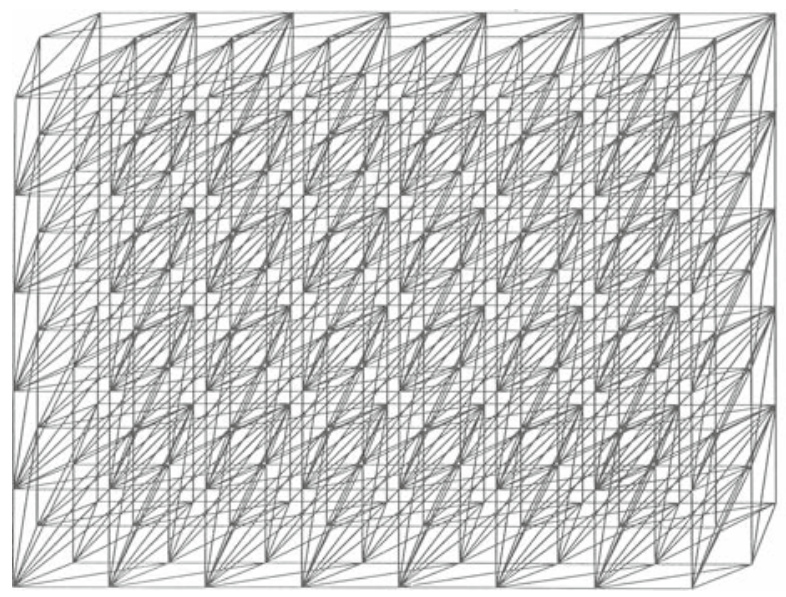

Fig. 8 Four-dimensional hypercubes divided into simplices and stacked to form a four-dimensional lattice

up to regular contributions (i.e., constant terms in the vicinity of $k_{c}$ ). Similarly one has for the curvature fluctuation

$$
\chi_{\mathcal{R}}(k) \underset{k \rightarrow k_{c}}{\sim} A_{\chi_{\mathcal{R}}}\left|k_{c}-k\right|^{-(2-d v)}
$$

At a critical point the fluctuation $\chi$ is in general expected to diverge, corresponding to the presence of a divergent correlation length. From such averages one can therefore in principle extract the correlation length exponent $v$ of (192) without having to compute a correlation function.

As far as the lattice is concerned, one starts for example with the 4-d hypercube of Fig. 5 divided into simplices, and then stacks a number of such cubes in such a way as to construct an arbitrarily large lattice, as shown in Fig. 8. Other lattice structures are of course possible, including even a random lattice. The expectation is that for long range correlations involving distance scales much larger than the lattice spacing the precise structure of the underlying lattice structure will not matter. This expectation of the existence of a unique scaling limit is known as universality of critical behavior.

Typically the lattice sizes investigated range from $4^{4}$ sites $\left(3,840\right.$ edges) to $32^{4}$ sites $(15,728,640$ edges). On a dedicated massively parallel supercomputer millions of consecutive edge length configurations can be generated for tens of values of $k$ in a few day's or week's time. Furthermore the bare cosmological constant $\lambda_{0}$ appearing in the gravitational action of (96) can be fixed at 1 in units of the cutoff, since it just sets the overall length scale in the problem. The higher derivative coupling $a$ can be set to a value very close to 0 since one ultimately is interested in the limit $a \rightarrow 0$, corresponding to the pure Einstein theory.

One finds that for the measure in (95) this choice of parameters leads to a well behaved ground state for $k<k_{c}$ for higher derivative coupling $a \rightarrow 0$. The system then resides in the "smooth" phase, with an effective dimensionality close to four. On 

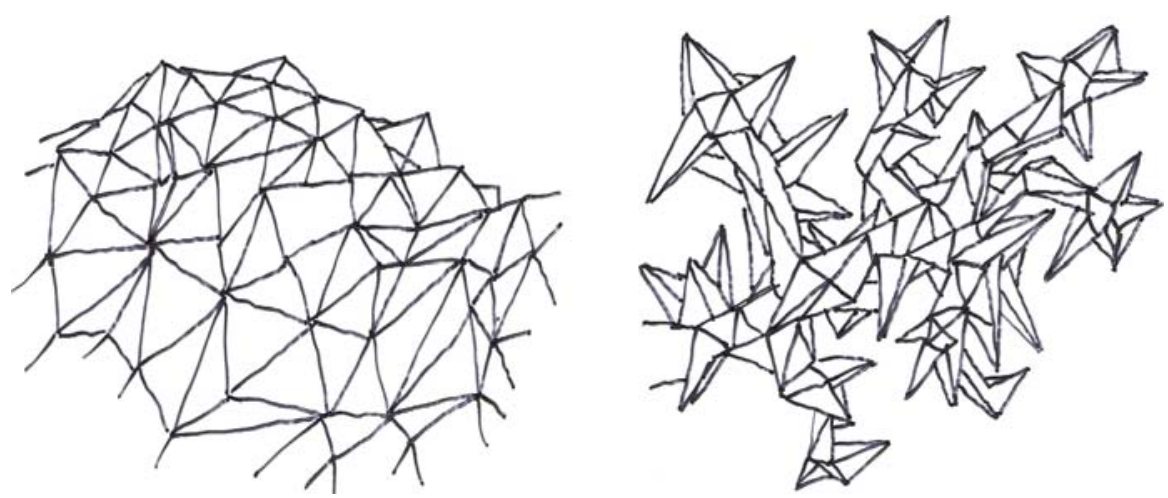

Fig. 9 A pictorial description of the smooth (left) and rough (right) phases of four-dimensional lattice quantum gravity

the other hand for $k>k_{c}$ the curvature becomes very large and the lattice collapses into degenerate configurations with very long, elongated simplices (see Fig. 9).

One finds that as $k$ is varied, the average curvature $\mathcal{R}$ is negative for sufficiently small $k$ ("smooth" phase), and appears to go to zero continuously at some finite value $k_{c}$. For $k>k_{c}$ the curvature becomes very large, and the simplices tend to collapse into degenerate configurations with very small volumes $\left(\langle V\rangle /\left\langle l^{2}\right\rangle^{2} \sim 0\right)$. This "rough" or "collapsed" phase is the region of the usual weak field expansion $(G \rightarrow 0)$. In this phase the lattice collapses into degenerate configurations with very long, elongated simplices. This phenomenon is usually interpreted as a lattice remnant of the conformal mode instability of Euclidean gravity discussed earlier.

There are a number of ways by which the critical exponents can be determined accurately from numerical simulations, but it is beyond the scope of this review to go into details. For example, one way to extract the critical exponent $v$ is to fit the average curvature to the form [see (195)]

$$
\mathcal{R}(k) \underset{k \rightarrow k_{c}}{\sim}-A_{\mathcal{R}}\left(k_{c}-k\right)^{\delta} .
$$

Using this general set of procedures one obtains eventually

$$
k_{c}=0.0636(11) \quad v=0.335(9) \text {, }
$$

which suggests $v=1 / 3$ for pure quantum gravity. Note that at the critical point the gravitational coupling is not weak, $G_{c} \approx 0.626$ in units of the ultraviolet cutoff.

Often it can be advantageous to express results obtained in the cutoff theory in terms of physical (i.e., cutoff independent) quantities. By the latter one means quantities for which the cutoff dependence has been re-absorbed, or restored, in the relevant definition. As an example, an expression equivalent to (195), relating the vacuum 
Table 1 Direct determinations of the critical exponent $v^{-1}$ for quantum gravitation, using various analytical and numerical methods in three and four space-time dimensions

\begin{tabular}{lll}
\hline Method & $v^{-1}$ in $d=3$ & $v^{-1}$ in $d=4$ \\
\hline Lattice & $1.67(6)$ & - \\
Lattice & - & $2.98(7)$ \\
$2+\epsilon$ & 1.6 & 4.4 \\
Truncation & 1.2 & 2.666 \\
Exact? & 1.5882 & 3 \\
\hline
\end{tabular}

expectation value of the local scalar curvature to the physical correlation length $\xi$, is

$$
\frac{\left\langle\int d x \sqrt{g} R(x)\right\rangle}{\left\langle\int d x \sqrt{g}\right\rangle} \underset{G \rightarrow G_{c}}{\sim} \text { const. }\left(l_{P}^{2}\right)^{(d-2-1 / v) / 2}\left(\frac{1}{\xi^{2}}\right)^{(d-1 / v) / 2},
$$

which is obtained by substituting (192) into (195). The correct dimensions have been restored in this last equation by supplying appropriate powers of the Planck length $l_{P}=G_{\text {phys }}^{1 /(d-2)}$, which involves the ultraviolet cutoff $\Lambda$. Then for $v=1 / 3$ the result of (199) becomes particularly simple

$$
\frac{\left\langle\int d x \sqrt{g} R(x)\right\rangle}{\left\langle\int d x \sqrt{g}\right\rangle} \underset{G \rightarrow G_{c}}{\sim} \text { const. } \frac{1}{l_{P} \xi}
$$

Note that a naive estimate based on dimensional arguments would have suggested the incorrect result $\sim 1 / l_{P}^{2}$. Instead the above expression actually vanishes at the critical point. This shows that $v$ plays the role of an anomalous dimension, determining the magnitude of deviations from naive dimensional arguments. It is amusing to note that the value $v=1 / 3$ for gravity does not correspond to any known field theory or statistical mechanics model in four dimensions. For a perhaps related system, namely dilute branched polymers, it is known that $v=1 / 2$ in three dimensions, and $v=1 / 4$ at the upper critical dimension $d=8$, so one would expect a value close to $1 / 3$ somewhere in between. On the other hand for a scalar field one would have obtained $v=1$ in $d=2$ and $v=\frac{1}{2}$ for $d \geq 4$, which seems excluded.

Table 1 provides a summary of the critical exponents for quantum gravitation as obtained by various perturbative and non-perturbative methods in three and four dimensions. The $2+\epsilon$ and the truncation method results were discussed previously. The lattice model of (96) in four dimensions gives for the critical point $G_{c} \approx 0.626$ in units of the ultraviolet cutoff, and $v^{-1}=2.98(7)$ which is used for comparison in Table 1 . In three dimensions the numerical results are consistent with the universality class of the interacting scalar field.

\section{Renormalization group and lattice continuum limit}

The discussion in the previous sections points to the existence of a phase transition in the lattice gravity theory, with divergent correlation length in the vicinity of the 
critical point, as in (192)

$$
\xi(k) \underset{k \rightarrow k_{c}}{\sim} A_{\xi}\left|k_{c}-k\right|^{-v}
$$

One expects the scaling result of (201) close to the fixed point, which we choose to rewrite here in terms of the inverse correlation length $m \equiv 1 / \xi$

$$
m=\Lambda A_{m}\left|k-k_{c}\right|^{\nu}
$$

In the above expression the correct dimension for $m$ (inverse length) has been restored by inserting explicitly on the r.h.s. the ultraviolet cutoff $\Lambda$. Here $k$ and $k_{c}$ are of course still dimensionless quantities, and correspond to the bare microscopic couplings at the cutoff scale, $k \equiv k(\Lambda) \equiv 1 /(8 \pi G(\Lambda))$. $A_{m}$ is a calculable numerical constant, related to $A_{\xi}$ in (192) by $A_{m}=A_{\xi}^{-1}$. It is worth pointing out that the above expression for $m(k)$ is almost identical in structure to the one for the non-linear $\sigma$-model in the $2+\epsilon$ expansion, (25) and in the large $N$ limit. It is of course also quite similar to $2+\epsilon$ result for continuum gravity, (50).

The lattice continuum limit corresponds to the large cutoff limit taken at fixed $m$ or $\xi$,

$$
\Lambda \rightarrow \infty, \quad k \rightarrow k_{c}, \quad m \text { fixed }
$$

which shows that the continuum limit is reached in the vicinity of the ultraviolet fixed point (see Fig. 10). Phrased equivalently, one takes the limit in which the lattice spacing $a \approx 1 / \Lambda$ is sent to zero at fixed $\xi=1 / m$, which requires an approach to the non-trivial UV fixed point $k \rightarrow k_{c}$. The quantity $\xi$ is supposed to be a renormalization group invariant, a physical scale independent of the scale at which the theory is probed. In practice, since the cutoff ultimately determines the physical value of Newton's constant $G, \Lambda$ cannot be taken to $\infty$. Instead a very large value will suffice, $\Lambda^{-1} \sim 10^{-33} \mathrm{~cm}$, for which it will still be true that $\xi \gg \Lambda$ which is all that is required for the continuum limit.

For discussing the renormalization group behavior of the coupling it will be more convenient to write the result of (202) directly in terms of Newton's constant $G$ as

$$
m=\Lambda\left(\frac{1}{a_{0}}\right)^{v}\left[\frac{G(\Lambda)}{G_{c}}-1\right]^{\nu},
$$

with the dimensionless constant $a_{0}$ related to $A_{m}$ by $A_{m}=1 /\left(a_{0} k_{c}\right)^{v}$. Note that the above expression only involves the dimensionless ratio $G(\Lambda) / G_{c}$, which is the only relevant quantity here. From the knowledge of the dimensionless constant $A_{m}$ in (202) one can estimate from first principles the value of $a_{0}$ in (209). Lattice results for the correlation functions at fixed geodesic distance give a value for $A_{m} \approx 0.72$ with a significant uncertainty, which, when combined with the values $k_{c} \simeq 0.0636$ and $v \simeq 0.335$ given above, gives $a_{0}=1 /\left(k_{c} A_{m}^{1 / v}\right) \simeq 42$. 

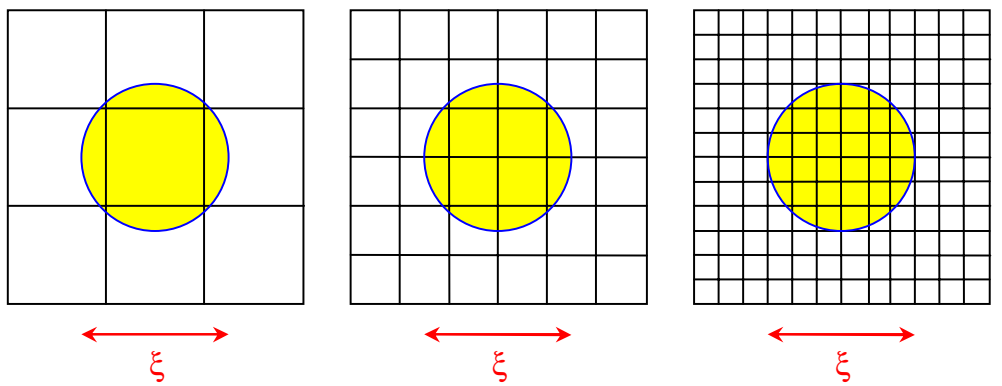

Fig. 10 The lattice quantum continuum limit is gradually approached by considering sequences of lattices with increasingly larger correlation lengths $\xi$ in lattice units. Such a limit requires the existence of an ultraviolet fixed point, where quantum field correlations extend over many lattice spacing

The renormalization group invariance of the physical quantity $m$ requires that the running gravitational coupling $G(\mu)$ varies in the vicinity of the fixed point in accordance with the above equation, with $\Lambda \rightarrow \mu$, where $\mu$ is now an arbitrary scale,

$$
m=\mu\left(\frac{1}{a_{0}}\right)^{v}\left[\frac{G(\mu)}{G_{c}}-1\right]^{v},
$$

The latter is equivalent to the renormalization group invariance requirement

$$
\mu \frac{d}{d \mu} m(\mu, G(\mu))=0
$$

provided $G(\mu)$ is varied in a specific way. (206) can therefore be used to obtain, if one so wishes, $a \beta$-function for the coupling $G(\mu)$ in units of the ultraviolet cutoff,

$$
\mu \frac{\partial}{\partial \mu} G(\mu)=\beta(G(\mu))
$$

with $\beta(G)$ given in the vicinity of the non-trivial fixed point, using (205), by

$$
\beta(G) \equiv \mu \frac{\partial}{\partial \mu} G(\mu) \underset{G \rightarrow G_{c}}{\sim}-\frac{1}{v}\left(G-G_{c}\right)+\cdots .
$$

The above procedure is in fact in complete analogy to what is done for the non-linear $\sigma$-model. But the last two steps are not really necessary, for one can obtain the scale dependence of the gravitational coupling directly from (205), by simply solving for $G(\mu)$

$$
G(\mu)=G_{c}\left[1+a_{0}\left(m^{2} / \mu^{2}\right)^{1 / 2 v}+\cdots\right]
$$

This last expression can be compared directly to the $2+\epsilon$ result of (46), as well as to the $\sigma$-model result of (22). The physical dimensions of $G$ can be restored by multiplying the above expression on both sides by the ultraviolet cutoff $\Lambda$, if one so desires. 
One concludes that the above result physically implies gravitational anti-screening: the gravitational coupling $G$ increases with distance. In conclusion, the lattice result for $G(\mu)$ in (209) and the $\beta$-function in (208) are qualitatively similar to what one finds both in the $2+\epsilon$ expansion for gravity and in the non-linear $\sigma$-model in the strong coupling phase.

\section{Curvature scales and gravitational condensate}

As can be seen from (30) the path integral for pure quantum gravity can be made to depend on the gravitational coupling $G$ and the cutoff $\Lambda$ only: by a suitable rescaling of the metric, or the edge lengths in the discrete case, one can set the cosmological constant to unity in units of the cutoff. The remaining coupling $G$ should then be viewed more appropriately as the gravitational constant in units of the cosmological constant $\lambda$.

The renormalization group running of $G(\mu)$ in (209) involves an invariant scale $\xi=1 / m$. At first it would seem that this scale could take any value, including very small ones based on the naive estimate $\xi \sim l_{P}$, which would preclude any observable quantum effects in the foreseeable future. But the result of (199) and (200) suggest otherwise, namely that the non-perturbative scale $\xi$ is in fact related to curvature. From astrophysical observation the average curvature is very small, so one would conclude from (200) that $\xi$ is very large, and possibly macroscopic. But the problem with (200) is that it involves the lattice Ricci scalar, a quantity related curvature probed by parallel transporting vectors around infinitesimal loops with size comparable to the lattice cutoff $\Lambda^{-1}$. What one would like is instead a relationship between $\xi$ and quantities which describe the geometry on larger scales.

In many ways the quantity $m$ of (205) behaves as a dynamically generated mass scale, quite similar to what happens in the non-linear $\sigma$-model case, or in the $2+\epsilon$ gravity case (47). From the classical field equation $R=4 \lambda$ one can relate the above $\lambda$, and therefore the mass-like parameter $m$, to curvature, which leads to the identification

$$
\lambda_{\mathrm{obs}} \simeq \frac{1}{\xi^{2}}
$$

with $\lambda_{\text {obs }}$ the observed small but non-vanishing cosmological constant.

A further indication that the identification of the observed scaled cosmological constant with a mass-like, and therefore renormalization group invariant, term makes sense beyond the weak field limit can be seen for example by comparing the structure of the three classical field equations

$$
\begin{aligned}
R_{\mu \nu}-\frac{1}{2} g_{\mu \nu} R+\lambda g_{\mu \nu} & =8 \pi G T_{\mu \nu} \\
\partial^{\mu} F_{\mu \nu}+\mu^{2} A_{\nu} & =4 \pi e j_{v} \\
\partial^{\mu} \partial_{\mu} \phi+m^{2} \phi & =\frac{g}{3 !} \phi^{3}
\end{aligned}
$$


for gravity, QED (massive via the Higgs mechanism) and a self-interacting scalar field, respectively.

A third argument suggesting the identification of the scale $\xi$ with large scale curvature, and therefore with the observed scaled cosmological constant, goes as follows. Observationally the curvature on large scale can be determined by parallel transporting vectors around very large loops, with typical size much larger than the lattice cutoff $l_{P}$. In gravity, curvature is detected by parallel transporting vectors around closed loops. This requires the calculation of a path dependent product of Lorentz rotations $\mathbf{R}$, in the Euclidean case elements of $\mathrm{SO}(4)$, as discussed earlier. From it then follows the identification of the correlation length $\xi$ with a measure of large scale curvature, the most natural candidate being the scaled cosmological constant $\lambda_{\text {phys }}$, as in (210). This relationship, taken at face value, implies a very large, cosmological value for $\xi \sim 10^{28} \mathrm{~cm}$, given the present bounds on $\lambda_{\text {phys. }}$ Thus the modified Einstein equations, incorporating the quantum running of $G$, should read

$$
R_{\mu \nu}-\frac{1}{2} g_{\mu \nu} R+\lambda g_{\mu \nu}=8 \pi G(\mu) T_{\mu \nu}
$$

with $\lambda \simeq \frac{1}{\xi^{2}}$. Here only $G(\mu)$ on the r.h.s. scale-dependent in accordance with (209). The precise meaning of $G(\mu)$ in a covariant framework will be given shortly.

It is worth pointing out here that the gravitational vacuum condensate, which only exists in the strong coupling phase $G>G_{c}$, and which is proportional to the curvature, is genuinely non-perturbative. Thus one can summarize the result of (210) as

$$
\mathcal{R}_{\mathrm{obs}} \simeq\left(10^{-30} e V\right)^{2} \sim \xi^{-2}
$$

where the condensate is, according to (204), non-analytic at $G=G_{c}$. A graviton vacuum condensate of order $\xi^{-1}-10^{-30} \mathrm{eV}$ is of course extraordinarily small compared to the QCD color condensate $\left(\Lambda_{\overline{\mathrm{MS}}} \simeq 220 \mathrm{MeV}\right)$ and the electro-weak Higgs condensate $(v \simeq 250 \mathrm{GeV})$. One can pursue the analogy with non-Abelian gauge theories further by stating that the quantum gravity theory cannot provide a value for the nonperturbative curvature scale $\xi$ : it needs to be fixed by some sort of phenomenological input, either by (209) or by (210). But one important message is that the scale $\xi$ in those two equations is one and the same.

\section{Effective field equations}

To summarize the results of the previous section, the result of (209) implies for the running gravitational coupling in the vicinity of the ultraviolet fixed point

$$
G\left(k^{2}\right)=G_{c}\left[1+a_{0}\left(\frac{m^{2}}{k^{2}}\right)^{\frac{1}{2 v}}+O\left(\left(m^{2} / k^{2}\right)^{\frac{1}{v}}\right)\right]
$$

with $m=1 / \xi, a_{0}>0$ and $v \simeq 1 / 3$. Since $\xi$ is expected to be very large, the quantity $G_{c}$ in the above expression should now be identified with the laboratory scale value 
$\sqrt{G_{c}} \sim 1.6 \times 10^{-33} \mathrm{~cm}$. The effective interaction in real space is often obtained by Fourier transform, but the above expression is a bit singular as $k^{2} \rightarrow 0$. The infrared divergence needs to be regulated, which can be achieved by utilizing as the lower limit of momentum integration $m=1 / \xi$. Alternatively, as a properly infrared regulated version of the above expression one can use

$$
G\left(k^{2}\right) \simeq G_{c}\left[1+a_{0}\left(\frac{m^{2}}{k^{2}+m^{2}}\right)^{\frac{1}{2 v}}+\cdots\right]
$$

Then at very large distances $r \gg \xi$ the gravitational coupling approaches the finite value $G_{\infty}=\left(1+a_{0}+\cdots\right) G_{c}$.

The first step in analyzing the consequences of a running of $G$ is to re-write the expression for $G\left(k^{2}\right)$ in a coordinate-independent way, for example by the use of a nonlocal Vilkovisky-type effective actions. Since in going from momentum to position space one usually employs $k^{2} \rightarrow-\square$, to obtain a quantum-mechanical running of the gravitational coupling one needs to make the replacement

$$
G \rightarrow G(\square)
$$

and therefore from (214)

$$
G(\square)=G_{c}\left[1+a_{0}\left(\frac{1}{\xi^{2} \square}\right)^{\frac{1}{2 v}}+\cdots\right] .
$$

The running of $G$ is expected to lead to a non-local gravitational action, for example of the form

$$
I=\frac{1}{16 \pi G} \int d x \sqrt{g}\left(1-a_{0}\left[\frac{1}{\xi^{2} \square}\right)^{1 / 2 v}+\cdots\right] R .
$$

Due to the fractional exponent in general the covariant operator appearing in the above expression, namely

$$
A(\square)=a_{0}\left(\frac{1}{\xi^{2} \square}\right)^{1 / 2 v}
$$

has to be suitably defined by analytic continuation from positive integer powers. The latter can be done, for example, by computing $\square^{n}$ for positive integer $n$ and then analytically continuing to $n \rightarrow-1 / 2 v$.

Had one not considered the action of (218) as a starting point for constructing the effective theory, one would naturally be led [following (216)] to consider the following effective field equations

$$
R_{\mu \nu}-\frac{1}{2} g_{\mu \nu} R+\lambda g_{\mu \nu}=8 \pi G(1+A(\square)) T_{\mu \nu}
$$


the argument again being the replacement $G \rightarrow G(\square) \equiv G(1+A(\square))$. Being manifestly covariant, these expressions at least satisfy some of the requirements for a set of consistent field equations incorporating the running of $G$. The above effective field equation can in fact be re-cast in a form similar to the classical field equations

$$
R_{\mu \nu}-\frac{1}{2} g_{\mu \nu} R+\lambda g_{\mu \nu}=8 \pi G \tilde{T}_{\mu \nu}
$$

with $\tilde{T}_{\mu \nu}=(1+A(\square)) T_{\mu \nu}$ defined as an effective, or gravitationally dressed, energymomentum tensor. Just like the ordinary Einstein gravity case, in general $\tilde{T}_{\mu \nu}$ might not be covariantly conserved a priori, $\nabla^{\mu} \tilde{T}_{\mu \nu} \neq 0$, but ultimately the consistency of the effective field equations demands that it be exactly conserved, in consideration of the Bianchi identity satisfied by the Riemann tensor. In a sense the running of $G$ can be interpreted as due to some sort of "vacuum fluid", introduced to account for the vacuum polarization contribution, whose energy momentum tensor one would expect to be ultimately covariantly conserved. That the procedure is consistent in general is not entirely clear, in which case the present approach should perhaps be limited to phenomenological considerations.

\section{Static isotropic solution}

One can show that the quantum correction due to the running of $G$ can be described, at least in the non-relativistic limit of (215) as applied to Poisson's equation, in terms of a vacuum energy density $\rho_{m}(r)$, distributed around the static source of strength $M$ in accordance with the result

$$
\rho_{m}(r)=\frac{1}{8 \pi} c_{\nu} a_{0} M m^{3}(m r)^{-\frac{1}{2}\left(3-\frac{1}{v}\right)} K_{\frac{1}{2}\left(3-\frac{1}{v}\right)}(m r)
$$

with a constant

$$
c_{v} \equiv \frac{2^{\frac{1}{2}\left(5-\frac{1}{v}\right)}}{\sqrt{\pi} \Gamma\left(\frac{1}{2 v}\right)} .
$$

and such that

$$
4 \pi \int_{0}^{\infty} r^{2} d r \rho_{m}(r)=a_{0} M
$$

In the relativistic context, a manifestly covariant implementation of the running of $G$, via the $G(\square)$ given in (217), will induce a non-vanishing effective pressure term. It is natural therefore to attempt to represent the vacuum polarization cloud by a relativistic perfect fluid, with energy-momentum tensor $T_{\mu \nu}=(p+\rho) u_{\mu} u_{\nu}+g_{\mu \nu} p$. 
Solving the resulting field equations gives a solution only for $v=1 / 3$ and one finds

$$
A^{-1}(r)==B(r)=1-\frac{2 M G}{r}+\frac{4 a_{0} M G m^{3}}{3 \pi} r^{2} \ln (m r)+\cdots
$$

After a bit of work one can then obtain an expression for the effective pressure $p_{m}(r)$, and one finds again in the limit $r \gg 2 M G$

$$
p_{m}(r)=\frac{a_{0}}{2 \pi^{2}} M m^{3} \ln (m r)+\cdots
$$

The expressions for $A(r)$ and $B(r)$ are therefore consistent with a gradual slow increase in $G$ with distance, in accordance with the formula

$$
G \rightarrow G(r)=G\left(1+\frac{a_{0}}{3 \pi} m^{3} r^{3} \ln \frac{1}{m^{2} r^{2}}+\cdots\right)
$$

in the regime $r \gg 2 M G$, and therefore of course in agreement with the original result of (214) or (215), namely that the classical laboratory value of $G$ is obtained for $r \ll \xi$. There are similarities, as well as some rather substantial differences, with the corresponding QED small $r$ result

$$
Q \rightarrow Q(r)=Q\left(1+\frac{\alpha}{3 \pi} \ln \frac{1}{m^{2} r^{2}}+\cdots\right)
$$

In the gravity case, the correction vanishes as $r$ goes to zero: in this limit one is probing the bare mass, unobstructed by its virtual graviton cloud. In some ways the running $G$ term acts as a local cosmological constant term, for which the $r$ dependence of the vacuum solution for small $r$ is fixed by the nature of the Schwarzschild solution with a cosmological constant term. One can therefore wonder what these solutions might look like in $d$ dimensions, and after some straightforward calculations one finds that in $d \geq 4$ dimensions only $v=1 /(d-1)$ is possible.

\section{Cosmological solutions}

A scale dependent Newton's constant will lead to small modifications of the standard cosmological solutions to the Einstein field equations. Here we will provide a brief discussion of what modifications are expected from the effective field equations on the basis of $G(\square)$, as given in (216), which itself originates in (215) and (214). The starting point is the quantum effective field equations of (220),

$$
R_{\mu \nu}-\frac{1}{2} g_{\mu \nu} R+\lambda g_{\mu \nu}=8 \pi G(1+A(\square)) T_{\mu \nu}
$$


with $A(\square)$ defined in (219). In the Friedmann-Robertson-Walker (FRW) framework these are applied to the standard homogeneous isotropic metric

$$
d s^{2}=-d t^{2}+a^{2}(t)\left\{\frac{d r^{2}}{1-k r^{2}}+r^{2}\left(d \theta^{2}+\sin ^{2} \theta d \varphi^{2}\right)\right\}
$$

It should be noted that there are two quantum contributions to the above set of effective field equations. The first one arises because of the presence of a non-vanishing cosmological constant $\lambda \simeq 1 / \xi^{2}$ caused by the non-perturbative vacuum condensate of (210). As in the case of standard FRW cosmology, this is expected to be the dominant contributions at large times $t$, and gives an exponential (for $\lambda>0$ ) or cyclic (for $\lambda<0$ ) expansion of the scale factor. The second contribution arises because of the running of $G$ in the effective field equations,

$$
G(\square)=G(1+A(\square))=G\left[1+a_{0}\left(\xi^{2} \square\right)^{-\frac{1}{2 v}}+\cdots\right]
$$

for $t \ll \xi$, with $v \simeq 1 / 3$ and $a_{0}>0$ a calculable coefficient of order one [see (214), (215)]. The next step is to examine the full effective field equations with a cosmological constant $\lambda=0$,

$$
R_{\mu \nu}-\frac{1}{2} g_{\mu \nu} R=8 \pi G(1+A(\square)) T_{\mu \nu}
$$

Here the d'Alembertian operator

$$
\square=g^{\mu v} \nabla_{\mu} \nabla_{v}
$$

acts on a second rank tensor,

$$
\begin{gathered}
\nabla_{\nu} T_{\alpha \beta}=\partial_{\nu} T_{\alpha \beta}-\Gamma_{\alpha \nu}^{\lambda} T_{\lambda \beta}-\Gamma_{\beta \nu}^{\lambda} T_{\alpha \lambda} \equiv I_{\nu \alpha \beta} \\
\nabla_{\mu}\left(\nabla_{\nu} T_{\alpha \beta}\right)=\partial_{\mu} I_{\nu \alpha \beta}-\Gamma_{\nu \mu}^{\lambda} I_{\lambda \alpha \beta}-\Gamma_{\alpha \mu}^{\lambda} I_{\nu \lambda \beta}-\Gamma_{\beta \mu}^{\lambda} I_{\nu \alpha \lambda}
\end{gathered}
$$

Next one assumes again that $T_{\mu \nu}$ has the perfect fluid form, for which one obtains from the action of $\square$ on $T_{\mu \nu}$

$$
\begin{aligned}
\left(\square T_{\mu \nu}\right)_{t t} & =6[\rho(t)+p(t)]\left(\frac{\dot{a}(t)}{a(t)}\right)^{2}-3 \dot{\rho}(t) \frac{\dot{a}(t)}{a(t)}-\ddot{\rho}(t) \\
\left(\square T_{\mu \nu}\right)_{r r} & =\frac{1}{1-k r^{2}}\left\{2[\rho(t)+p(t)] \dot{a}(t)^{2}-3 \dot{p}(t) a(t) \dot{a}(t)-\ddot{p}(t) a(t)^{2}\right\} \\
\left(\square T_{\mu \nu}\right)_{\theta \theta} & =r^{2}\left(1-k r^{2}\right)\left(\square T_{\mu \nu}\right)_{r r} \\
\left(\square T_{\mu \nu}\right)_{\varphi \varphi} & =r^{2}\left(1-k r^{2}\right) \sin ^{2} \theta\left(\square T_{\mu \nu}\right)_{r r}
\end{aligned}
$$

with the remaining components equal to zero. Note that a non-vanishing pressure contribution is generated in the effective field equations, even if one assumes initially a 
pressureless fluid, $p(t)=0$. As before, repeated applications of the d'Alembertian $\square$ to the above expressions leads to rapidly escalating complexity, which can only be tamed by introducing some further simplifying assumptions, such as a power law behavior for the density, $\rho(t)=\rho_{0} t^{\beta}$, and $p(t)=0$. After a lengthy calculation one finds for a universe filled with non-relativistic matter $(p=0)$, the effective Friedmann equations then have the following appearance

$$
\begin{aligned}
\frac{k}{a^{2}(t)}+\frac{\dot{a}^{2}(t)}{a^{2}(t)} & =\frac{8 \pi G(t)}{3} \rho(t)+\frac{1}{3 \xi^{2}} \\
& =\frac{8 \pi G}{3}\left[1+c_{\xi}(t / \xi)^{1 / v}+\cdots\right] \rho(t)+\frac{1}{3} \lambda
\end{aligned}
$$

for the $t t$ field equation, and

$$
\frac{k}{a^{2}(t)}+\frac{\dot{a}^{2}(t)}{a^{2}(t)}+\frac{2 \ddot{a}(t)}{a(t)}=-\frac{8 \pi G}{3}\left[c_{\xi}(t / \xi)^{1 / v}+\cdots\right] \rho(t)+\lambda
$$

for the $r r$ field equation. The running of $G$ appropriate for the Robertson-Walker metric, and appearing explicitly in the first equation, is given by

$$
G(t)=G\left[1+c_{\xi}\left(\frac{t}{\xi}\right)^{1 / v}+\cdots\right]
$$

with $c_{\xi}$ of the same order as $a_{0}$ of (214). Note that the running of $G(t)$ induces as well an effective pressure term in the second $(r r)$ equation. We wish to emphasize that we are not talking here about models with a time-dependent value of $G$. Thus, for example, the value of $G \simeq G_{c}$ at laboratory scales should be taken to be constant throughout most of the evolution of the universe.

Finally it should be noted that the effective Friedmann equations of (236) and (237) also bear a superficial degree of resemblance to what might be obtained in some scalar-tensor theories of gravity, where the gravitational Lagrangian is postulated to be some singular function of the scalar curvature. The former scenario would then correspond the to an effective gravitational action

$$
I_{\mathrm{eff}} \simeq \frac{1}{16 \pi G} \int d x \sqrt{g}\left(R+\frac{f \xi^{-\frac{1}{v}}}{|R|^{\frac{1}{2 v}-1}}-2 \lambda\right)
$$

but with $v=1 / 3, f$ a numerical constant of order one, and $\lambda \simeq 1 / \xi^{2}$.

Acknowledgments The author wishes to thank Hermann Nicolai, Stefan Theisen and the Max Planck Institut für Gravitationsphysik (Albert-Einstein-Institut) in Potsdam for a very warm hospitality. This work was supported in part by the Max Planck Gesellschaft zur Förderung der Wissenschaften.

Open Access This article is distributed under the terms of the Creative Commons Attribution Noncommercial License which permits any noncommercial use, distribution, and reproduction in any medium, provided the original author(s) and source are credited. 


\section{References}

1. Aida, T., Kitazawa, Y.: Nucl. Phys. B491, 427 (1997)

2. Cheeger, J., Müller, W., Schrader, R.: Comm. Math. Phys. 92, 405 (1984)

3. Damour, T.: Experimental test of gravitational theory, in review of particle physics. J. Phys. G 33, $1(2006)$

4. DeWitt, B.S.: Phys. Rev. 162, 1195 (1967)

5. Faddeev, L., Popov, V.: Sov. Phys. Usp. 16, 777 (1974)

6. Faddeev, L., Popov, V.: Usp. Fiz. Nauk. 109, 427 (1974)

7. Feynman, R.P.: Lectures on Gravitation, 1962-1963. In: Morinigo, F.B., Wagner, W.G. (eds.) California Institute of Technology, Pasadena, 1971 (1962)

8. Feynman, R.P.: Acta Phys. Pol. 24, 697 (1963)

9. Hamber, H.W.: Quantum Gravitation-the Feynman Path Integral Approach. Springer, Berlin (2008)

10. Hamber, H.W.: Phys. Rev. D45, 507 (1992)

11. Hamber, H.W.: Nucl. Phys. B 400, 347 (1992)

12. Hamber, H.W., Williams, R.M.: Nucl. Phys. B 435, 361 (1995)

13. Hamber, H.W., Williams, R.M.: Phys. Rev. D 72, 044026; 75, 084014; 76, 084008 (2005)

14. Hartle, J.B.: J. Math. Phys. 26, 804 (1985)

15. Hartle, J.B., Hawking, S.W.: Phys. Rev. D 28, 2960 (1983)

16. Hawking, S.W.: In: Hawking, S.W., Israel, W. (eds.) General Relativity—an Einstein Centenary Survey. Cambridge University Press, London (1979)

17. Itzykson, C.: Fields on a random lattice. In: Progress in Gauge Field Theory. Cargése, France, 1-15 Sept. (1983)

18. Kawai, H., Kitazawa, Y., Ninomiya, M.: Nucl. Phys. B393, 280; B404 684 (1993)

19. Lee, T.D.: In Discrete Mechanics, 1983 Erice International School of Subnuclear Physics, vol. 21. Plenum Press, New York, 1985 (1983)

20. Leutwyler, H.: Phys. Rev. 134, 1155 (1964)

21. Misner, C.W.: Rev. Mod. Phys. 29, 497 (1957)

22. Modanese, G.: Phys. Rev. D 47, 502; 49, 6534 (1993)

23. Parisi, G.: On nonrenormalizable interactions. In: The Proceedings of the 1976 Cargése Summer Institute (1976)

24. Regge, T.: Nuovo Cimento 19, 558 (1961)

25. Regge, T., Williams, R.M.: J. Math. Phys. 41, 3964 (2000)

26. Reuter, M.: In: Nicolai, H. (ed.) Proceedings of the Conference Quantum Gravity: Challenges and Perspectives. Bad Honneff, Class. Quant. Grav. vol. xx (2008)

27. Symanzik, K.: Euclidean quantum field theory. In: Jost, R. (ed.) Varenna Lectures. Academic Press, New York (1969)

28. 't Hooft, G., Veltman, M.: Ann. Inst. Poincaré 20, 69 (1974)

29. Weinberg S.: Ultraviolet divergences in quantum gravity. In: Hawking, S.W., Israel, W. (eds.) General Relativity — an Einstein Centenary Survey. Cambridge University Press, London (1979)

30. Wilson, K.G.: Phys. Rev. Lett. 28, 548 (1972)

31. Zinn-Justin, J.: Quantum Field Theory and Critical Phenomena, 4th edn. Oxford University Press, New York (2002) 